ESAIM: COCV 20 (2014) 1059-1077

DOI: $10.1051 / \mathrm{cocv} / 2014007$
ESAIM: Control, Optimisation and Calculus of Variations

www.esaim-cocv.org

\title{
GROUND STATES OF SINGULARLY PERTURBED CONVECTION-DIFFUSION EQUATION WITH OSCILLATING COEFFICIENTS
}

\author{
A. Piatnitski ${ }^{1}$, A. Rybalko ${ }^{2}$ And V. Rybalko ${ }^{3}$
}

\begin{abstract}
We study the first eigenpair of a Dirichlet spectral problem for singularly perturbed convection-diffusion operators with oscillating locally periodic coefficients. It follows from the results of [A. Piatnitski and V. Rybalko, On the first eigenpair of singularly perturbed operators with oscillating coefficients. Preprint www.arxiv.org, arXiv:1206.3754] that the first eigenvalue remains bounded only if the integral curves of the so-called effective drift have a nonempty $\omega$-limit set. Here we consider the case when the integral curves can have both hyperbolic fixed points and hyperbolic limit cycles. One of the main goals of this work is to determine a fixed point or a limit cycle responsible for the first eigenpair asymptotics. Here we focus on the case of limit cycles that was left open in [A. Piatnitski and V. Rybalko, Preprint.
\end{abstract}

Mathematics Subject Classification. 35B27, 35B40.

Received March 19, 2013. Revised November 16, 2013.

Published online August 4, 2014.

\section{INTRODUCTION}

In this work we study the effect of slow and fast oscillations in the singularly perturbed spectral problem in a smooth bounded domain $\Omega \subset \mathbb{R}^{N}$. This problem reads

$$
\varepsilon a^{i j}\left(x, \frac{x}{\varepsilon}\right) \frac{\partial^{2} u}{\partial x_{i} \partial x_{j}}+b^{j}\left(x, \frac{x}{\varepsilon}\right) \frac{\partial u}{\partial x_{j}}+c\left(x, \frac{x}{\varepsilon}\right) u=\lambda u \quad \text { in } \Omega
$$

with the Dirichlet condition $u=0$ on $\partial \Omega$. We assume that the coefficients in (1.1) are sufficiently smooth (of the class $C^{2}\left(\bar{\Omega} \times \mathbb{R}^{N}\right)$ ) functions $Y$-periodic in the second variable, $Y=(0,1)^{N}$ being the periodicity cell; and $a^{i j}$ satisfy the symmetry and the uniform ellipticity assumptions. According to the Krein-Rutman theorem the first eigenvalue $\lambda_{\varepsilon}$ (the eigenvalue with the maximal real part) is real and simple, the corresponding eigenfunction $u_{\varepsilon}$ can be chosen to satisfy $0<u_{\varepsilon} \leq \max u_{\varepsilon}=1$ in $\Omega$. We are interested in the asymptotic behavior of $\lambda_{\varepsilon}$ and $u_{\varepsilon}$ as $\varepsilon \rightarrow 0$.

\footnotetext{
Keywords and phrases. Singularly perturbed operators, eigenpair asymptotics, homogenization.

1 Narvik University College, Postboks 385, 8505 Narvik, Norway, and P.N. Lebedev Physical Institute of RAS, 53, Leninski pr., 119991 Moscow, Russia. andrey@sci.lebedev.ru

2 Kharkiv National University of Economics, 9a Lenin ave., 61166 Kharkiv, Ukraine. n_rybalko@yahoo.com

3 Mathematical Department, B.Verkin Institute for Low Temperature Physics and Engineering of the NASU, 47 Lenin ave., 61103 Kharkiv, Ukraine, vrybalko@ilt.kharkov.ua
} 
Previously, spectral problems for self-adjoint elliptic operators with large potential and locally periodic oscillating coefficients were studied in [3]. It was shown that under natural assumptions the eigenfunctions are localized in the scale $\sqrt{\varepsilon}$ and admit factorization in this scale. The factorization method was also used in [7] for homogenization of the Dirichlet spectral problem for non-self adjoint operators with purely periodic coefficients, in [2] for weakly coupled systems and in [4] for studying a singularly perturbed non-stationary convectiondiffusion equation in a bounded domain. Similar parabolic problem in the whole space was considered in $[5,9]$, in this case factorization leads to homogenization in moving coordinates.

Two approaches were efficiently used for studying boundary value and spectral problems for singularly perturbed convection-diffusion operator. The first one relies on the probabilistic interpretation of the corresponding convection-diffusion process with small diffusion. This approach was developed in [19] and then applied in $[10,12]$ and other works.

Another approach is based on the viscosity solutions techniques for a singularly perturbed Hamilton-Jacobi PDE. In this approach one first makes a logarithmic transformation of a solution (a version of the so-called WKB method) and then studies the obtained singularly perturbed first-order nonlinear equation. This approach applied successfully in [14] and then was followed in many studies of singularly perturbed problems. Further discussion on the existing methods and results can be found in the companion paper [13].

It was shown in [13] that in the limit $\varepsilon \rightarrow 0$ problem (1.1) leads to an additive eigenvalue problem for an effective Hamilton-Jacobi equation. Namely, let us introduce the scaled logarithmic transformation $W_{\varepsilon}:=$ $-\varepsilon \log u_{\varepsilon}$ of the first eigenfunction $u_{\varepsilon}$, which satisfies

$$
-\varepsilon a^{i j}\left(x, \frac{x}{\varepsilon}\right) \frac{\partial^{2} W_{\varepsilon}}{\partial x_{i} \partial x_{j}}+H\left(\nabla W_{\varepsilon}, x, x / \varepsilon\right)+\varepsilon c\left(x, \frac{x}{\varepsilon}\right)=\varepsilon \lambda_{\varepsilon},
$$

where the Hamiltonian $H$ is given by $H(p, x, y)=a^{i j}(x, y) p_{i} p_{j}-b^{j}(x, y) p_{j}$. Then by Theorem 1 of [13] (with minor modifications) we have, $\varepsilon \lambda_{\varepsilon} \rightarrow \lambda_{\bar{H}}$ and (up to extracting a subsequence) $W_{\varepsilon} \rightarrow W$, where every limit pair $\left(\lambda_{\bar{H}}, W\right)$ is a viscosity solution of the additive eigenvalue problem with state constraint boundary conditions,

$$
\bar{H}(\nabla W, x)=\lambda_{\bar{H}} \quad \text { in } \Omega, \quad \bar{H}(\nabla W, x) \geq \lambda_{\bar{H}} \quad \text { on } \partial \Omega .
$$

We refer the interested reader to $[8,16]$, where the qualitative theory of problem $(1.3)$ can be found. The effective Hamiltonian $\bar{H}$ appearing in (1.3) is given as the additive eigenvalue of the problem

$$
-a^{i j}(x, y) \frac{\partial^{2} \theta}{\partial y_{i} \partial y_{j}}+H\left(p+\nabla_{y} \theta, x, y\right)=\bar{H}(p, x)
$$

with periodic boundary conditions, or, equivalently $\bar{H}(p, x)$ is the first eigenvalue (eigenvalue with the maximal real part) of the periodic linear eigenvalue problem

$$
-a^{i j}(x, y) \frac{\partial^{2} \vartheta}{\partial y_{i} \partial y_{j}}+\left(b^{j}(x, y)-2 a^{i j}(x, y) p_{i}\right) \frac{\partial \vartheta}{\partial y_{j}}+H(p, x, y) \vartheta=\bar{H}(p, x) \vartheta
$$

note also that $\theta=\log \vartheta$.

As discussed in [13] the limiting problem (1.3) has a unique eigenvalue $\lambda_{\bar{H}}$, but can have, in general, many eigenfunctions $W$. The uniqueness/nonuniqueness issue is intimately related to the structure of the so-called Aubry set $\mathcal{A}_{\bar{H}-\lambda_{\bar{H}}}$ which plays the role of a hidden boundary in (1.3), see, e.g., [16]. In the case $\lambda_{\bar{H}}=0$ the structure of the Aubry set is determined by the ODE $\dot{x}=-\bar{b}(x)$, the $\bar{b}(x)$ being the effective drift field defined as follows. Let $\theta^{*}(x, y)$ be the $Y$-periodic solution of

$$
\frac{\partial^{2}}{\partial y_{i} \partial y_{j}}\left(a^{i j}(x, y) \theta^{*}\right)-\frac{\partial}{\partial y_{j}}\left(b^{j}(x, y) \theta^{*}\right)=0
$$


normalized by

$$
\int_{Y} \theta^{*}(x, y) \mathrm{d} y=1
$$

Then

$$
\bar{b}^{j}(x)=\int_{Y} b^{j}(x, y) \theta^{*}(x, y) \mathrm{d} y .
$$

In this work we assume that

$\lambda_{\bar{H}}=0$, and the Aubry set $\mathcal{A}_{\bar{H}}$ is formed by a finite number of hyperbolic

fixed points and hyperbolic limit cycles of the ODE $\dot{x}=-\bar{b}(x)$, that are situated in the interior of $\Omega$.

The case when the equation $\dot{x}=-\bar{b}(x)$ does not have limit cycles in $\bar{\Omega}$ and the zero order term $c=0$ was treated in [13], while the present work focuses on the case of hyperbolic limit cycles.

The main result of this paper is

Theorem 1.1. Under condition (1.9) together with the above assumptions on the coefficients in (1.1) we have (i)

$$
\lim _{\varepsilon \rightarrow 0} \lambda_{\varepsilon}=\bar{\sigma}:=\max \left\{\sigma_{1}(\xi)+\sigma_{2}(\xi) ; \xi \in \mathcal{A}_{\bar{H}}\right\},
$$

where $\sigma_{1}(\xi), \sigma_{2}(\xi)$ are defined as follows, depending on whether $\xi$ is a fixed point of the ODE $\dot{x}=-\bar{b}(x)$ or $\xi$ is situated on a limit cycle of this ODE. If $\xi$ is a zero of $\bar{b}$ (fixed point of the ODE) then $\sigma_{1}(\xi)$ is the sum of negative real parts of the eigenvalues of the matrix

$$
\left(-\frac{\partial \bar{b}^{i}}{\partial x_{j}}(\xi)\right)_{i, j=\overline{1, N}},
$$

and

$$
\sigma_{2}(\xi)=\int_{Y} c(\xi, y) \theta^{*}(\xi, y) \mathrm{d} y
$$

If $\xi$ lies on a limit cycle then

$$
\sigma_{1}(\xi)=\frac{1}{P} \sum_{\left|\Lambda_{k}(\xi)\right|<1} \log \left|\Lambda_{k}(\xi)\right|,
$$

where $P>0$ is the minimal period of the cycle, and $\Lambda_{k}(\xi)$ are the eigenvalues of the linearized Poincaré map such that $\left|\Lambda_{k}(\xi)\right|<1$; in this case $\sigma_{2}(\xi)$ is given by

$$
\sigma_{2}(\xi)=\frac{1}{P} \int_{0}^{P} \int_{Y} c(x(t), y) \theta^{*}(x(t), y) \mathrm{d} y \mathrm{~d} t \quad \text { with } x(t) \text { solving } \quad \dot{x}=-\bar{b}(x), x(0)=\xi .
$$

(ii) Moreover, if the maximum in (1.10) is attained at exactly one connected component of the Aubry set (fixed point or limit cycle) then $W_{\varepsilon}$ converge uniformly on compacts in $\Omega$ to the maximal viscosity solution of (1.3) vanishing on the aforementioned component of the Aubry set.

Remark 1.2. In Theorem 1.1, $\Lambda_{k}(\xi)$ denote eigenvalues of a linearized Poincaré map corresponding to a point $\xi$ on the limit cycle and a transversal space hyperplane passing through this point. It is well-known however that the eigenvalues of the linearized Poincaré map depend neither on a particular choice of the point $\xi$ nor on a choice of the transversal hyperplane. Also remark that the hyperbolicity assumption imposed on a limit cycle means that the said eigenvalues do not lie on the unit circle. 
The approach in this work is an extension of that used in [13]. The main result is obtained combining local analysis on the scale $\sqrt{\varepsilon}$ in the vicinity of fixed points and limit cycles with the viscosity solutions type arguments. However the presence of limit cycles leads to new non-trivial difficulties. In particular, the local analysis near a fixed point $\xi$ heavily makes use of the fact that $\bar{b}(\xi)=0$ and thus has to be essentially modified in the case of a limit cycle. This analysis is not local any more and should take into account the behavior of the coefficients in the vicinity of the whole limit cycle. In order to treat limit cycles, we artificially introduce moving coordinates and study parabolic eigenvalue problems for the obtained operators. This method relies on qualitative results for parabolic Ornstein-Uhlenbeck type operators defined in the whole space.

The paper is organized as follows. In Section 2 we introduce moving coordinates and study blow up limits of the eigenfunctions of problem (1.1) and an auxiliary problem which lead to limiting parabolic spectral problems in the whole space. The latter problems are addressed in Section 3, where we establish the existence and uniqueness results within certain classes, moreover solutions of the said spectral problems are constructed via solutions of Riccati matrix equations. Section 4 completes the proof of Theorem 1.1. We construct there special subsolutions of (1.3) near limit cycles (this is done in the moving coordinates, like in Sect. 2), next we construct oscillating test functions to obtain bounds at infinity for blow up limits of the eigenfunctions. These bounds in turn allow us to identify uniquely the $\lim _{\varepsilon \rightarrow 0} \lambda_{\varepsilon}$ and select the $\lim _{\varepsilon \rightarrow 0} W_{\varepsilon}$ among solutions of (1.3). In Section 5 we obtain representation (1.11) for $\sigma_{1}(\xi)$ which is independent of a particular choice of local coordinates near the limit cycle. Section 6 presents an example. Finally the Appendix contains an existence and uniqueness result for periodic in time parabolic eigenvalue problems in the whole space.

\section{MOVING COORDINATES AND BLOW UP ANALYSIS}

In this section we obtain a lower bound for the principal eigenvalue $\lambda_{\varepsilon}$ and perform a blow up analysis in the vicinity of connected components of the Aubry set $\mathcal{A}_{\bar{H}}$. Under our standing assumptions, the Aubry set consists of fixed points and limit cycles of the ODE $\dot{x}=-\bar{b}(x)$. The study of fixed points is reduced to the adaptation of the corresponding results from [13]. Here we mostly focus on the case of limit cycles.

By a reason which will be clear later, it is convenient to transform (1.1) multiplying it by $\theta^{*}(x, x / \varepsilon)>0$, to the form

$$
\mathcal{L}_{\varepsilon} u=\lambda \theta^{*}(x, x / \varepsilon) u,
$$

where the coefficients of the operator $\mathcal{L}_{\varepsilon}$ are still denoted $a^{i j}(x, x / \varepsilon), b^{j}(x, x / \varepsilon)$ and $c(x, x / \varepsilon)$, and the following relation holds:

$$
\frac{\partial}{\partial y_{j}}\left(\frac{\partial}{\partial y_{i}} a^{i j}(x, y)-b^{j}(x, y)\right)=0 .
$$

Note that, due to (1.8) we now have

$$
\bar{b}^{j}(x)=\int_{Y} b^{j}(x, y) \mathrm{d} y .
$$

Assume that the ODE $\dot{x}=-\bar{b}(x)$ has a limit cycle $\mathcal{C} \subset \Omega$ with the minimal period $P>0$. That is, fixing a point $\xi_{0} \in \mathcal{C}$ and introducing the solution $\xi(t)$ of

$$
\dot{\xi}(t)=-\bar{b}(\xi(t)), \quad \xi(0)=\xi_{0},
$$

we have $\xi(t+P)=\xi(t)$ and $\mathcal{C}=\bigcup_{t \in[0, P)}\{\xi(t)\}$.

\subsection{Lower bound for eigenvalues}

To derive a lower bound for the first eigenvalue $\lambda_{\varepsilon}$ of $\mathcal{L}_{\varepsilon}$ consider, for a fixed $\delta>0$, the auxiliary operator

$$
\tilde{\mathcal{L}}_{\varepsilon} u=\varepsilon a^{i j}\left(x, \frac{x}{\varepsilon}\right) \frac{\partial^{2} u}{\partial x_{i} \partial x_{j}}+b^{j}\left(x, \frac{x}{\varepsilon}\right) \frac{\partial u}{\partial x_{j}}+c\left(x, \frac{x}{\varepsilon}\right) u-\delta \frac{\rho(x)}{\varepsilon} u \quad \text { in } \Omega
$$


with the Dirichlet condition $u=0$ on $\partial \Omega$, where $\rho$ is a smooth function such that $\rho>0$ in $\bar{\Omega} \backslash \mathcal{C}$, and $\rho(x)=\operatorname{dist}^{2}(x, \mathcal{C})$ in a neighborhood of $\mathcal{C}$. The reason behind introducing $\tilde{\mathcal{L}}_{\varepsilon}$ is that it has a better localized first eigenfunction. Moreover, due to the choice of the penalizing lower order term in (2.5) the eigenfunction localizes in the vicinity the curve $\mathcal{C}$.

Let $\tilde{\lambda}_{\varepsilon}$ be the first eigenvalue of the problem

$$
\tilde{\mathcal{L}}_{\varepsilon} u=\lambda \theta^{*}(x, x / \varepsilon) u .
$$

Since $\tilde{\mathcal{L}}_{\varepsilon} \phi(x) \leq \mathcal{L}_{\varepsilon} \phi(x)$ for every $\phi>0$, we have

$$
\tilde{\lambda}_{\varepsilon} \leq \lambda_{\varepsilon}
$$

because of the relations (see [17])

$$
\tilde{\lambda}_{\varepsilon}=\inf _{\phi} \sup _{x \in \Omega} \tilde{\mathcal{L}}_{\varepsilon} \phi(x) /\left(\theta^{*}(x, x / \varepsilon) \phi(x)\right) \quad \text { and } \quad \lambda_{\varepsilon}=\inf _{\phi} \sup _{x \in \Omega} \mathcal{L}_{\varepsilon} \phi(x) /\left(\theta^{*}(x, x / \varepsilon) \phi(x)\right),
$$

the infima being taken over $\phi \in C^{2}(\bar{\Omega}), \phi>0$ in $\Omega$ and $\phi=0$ on $\partial \Omega$.

Let $\tilde{u}_{\varepsilon}$ be the first eigenfunction of (2.6) normalized by $\tilde{u}_{\varepsilon}\left(\xi_{0}\right)=1$. We pass to the moving coordinate system $x^{\prime}=x-\xi(t)$. This change of coordinates transforms (2.6) into the parabolic PDE for $\tilde{U}_{\varepsilon}\left(t, x^{\prime}\right):=\tilde{u}_{\varepsilon}\left(x^{\prime}+\xi(t)\right)$,

$$
\begin{aligned}
&-\frac{\partial \tilde{U}_{\varepsilon}}{\partial t}+\varepsilon a_{\xi, \frac{\xi}{\varepsilon}}^{i j}\left(x^{\prime}, \frac{x^{\prime}}{\varepsilon}\right) \frac{\partial^{2} \tilde{U}_{\varepsilon}}{\partial x_{i}^{\prime} \partial x_{j}^{\prime}}+b_{\xi, \frac{\xi}{\varepsilon}}^{j}\left(x^{\prime}, \frac{x^{\prime}}{\varepsilon}\right) \frac{\partial \tilde{U}_{\varepsilon}}{\partial x_{j}^{\prime}} \\
&-\bar{b}^{j}(\xi(t)) \frac{\partial \tilde{U}_{\varepsilon}}{\partial x_{j}^{\prime}}+\left(c_{\xi, \frac{\xi}{\varepsilon}}\left(x^{\prime}, \frac{x^{\prime}}{\varepsilon}\right)-\frac{\delta}{\varepsilon} \rho\left(\xi(t)+x^{\prime}\right)\right) \tilde{U}_{\varepsilon}=\tilde{\lambda}_{\varepsilon} \theta_{\xi, \frac{\xi}{\varepsilon}}^{*}\left(x^{\prime}, \frac{x^{\prime}}{\varepsilon}\right) \tilde{U}_{\varepsilon}, \quad x^{\prime} \in \Omega-\xi(t),
\end{aligned}
$$

where we have used the pointwise equality

$$
\frac{\partial \tilde{U}_{\varepsilon}}{\partial t}=-\bar{b}^{j}(\xi(t)) \frac{\partial \tilde{U}_{\varepsilon}}{\partial x_{j}^{\prime}}
$$

which is due to the definition of $\tilde{U}_{\varepsilon}\left(t, x^{\prime}\right)$ and (2.4). The subscript " $\xi, \frac{\xi}{\varepsilon}$ " in (2.8) (and below) denotes the shift by $\xi(t)$ in $x$ and by $\xi(t) / \varepsilon$ in $y$, i.e., for instance, $a_{\xi, \frac{\xi}{\varepsilon}}^{i j}(x, y)=a^{i j}(x+\xi(t), y+\xi(t) / \varepsilon)$. Next, we want to rearrange the first and second order terms in equation (2.8) to make it more convenient for the further analysis. To this end we introduce $Y$-periodic (in $y$ ) solutions $T^{i j}$ of

$$
\frac{\partial T^{i j}}{\partial y_{i}}(x, y)=b^{j}(x, y)-\bar{b}^{j}(x)-\frac{\partial a^{i j}}{\partial y_{i}}(x, y), \quad T^{i j}=-T^{j i} .
$$

Thanks to (2.2) and (2.3) this equation has solutions $T^{i j}$ which are continuous and have bounded derivatives $\partial T^{i j} / \partial x_{k}$. Introducing the functions $q^{i j}(x, y)=a^{i j}(x, y)+T^{i j}(x, y), \tilde{q}^{j}=\partial q^{i j} / \partial x_{i}$ and considering (2.10), one can rewrite the first line of $(2.8)$ in the form

$$
-\frac{\partial \tilde{U}_{\varepsilon}}{\partial t}+\varepsilon \frac{\partial}{\partial x_{i}}\left(q_{\xi, \frac{\xi}{\varepsilon}}^{i j}\left(x^{\prime}, \frac{x^{\prime}}{\varepsilon}\right) \frac{\partial \tilde{U}_{\varepsilon}}{\partial x_{j}^{\prime}}\right)+\bar{b}^{j}\left(x^{\prime}+\xi(t)\right) \frac{\partial \tilde{U}_{\varepsilon}}{\partial x_{j}^{\prime}}-\varepsilon \tilde{q}_{\xi, \frac{\xi}{\varepsilon}}^{j}\left(x^{\prime}, \frac{x^{\prime}}{\varepsilon}\right) \frac{\partial \tilde{U}_{\varepsilon}}{\partial x_{j}^{\prime}} .
$$

Now set $z^{\prime}=x^{\prime} / \sqrt{\varepsilon}$ to find the equation for $\tilde{v}_{\varepsilon_{\left(t, z^{\prime}\right)}}=\tilde{U}_{\varepsilon}\left(t, \sqrt{\varepsilon} z^{\prime}\right)$,

$$
\begin{aligned}
& -\frac{\partial \tilde{v}_{\varepsilon}}{\partial t}+\frac{\partial}{\partial z_{i}^{\prime}}\left(a_{\varepsilon}^{i j}\left(t, z^{\prime}\right) \frac{\partial \tilde{v}_{\varepsilon}}{\partial z_{j}^{\prime}}\right)+h_{\varepsilon}^{j}\left(t, z^{\prime}\right) \frac{\partial \tilde{v}_{\varepsilon}}{\partial z_{j}^{\prime}} \\
& \quad+\left(c_{\xi, \frac{\xi}{\varepsilon}}\left(\sqrt{\varepsilon} z^{\prime}, \frac{z^{\prime}}{\sqrt{\varepsilon}}\right)-\delta \rho_{\varepsilon}\left(t, z^{\prime}\right)\right) \tilde{v}_{\varepsilon}=\tilde{\lambda}_{\varepsilon} \theta_{\xi, \frac{\xi}{\varepsilon}}^{*}\left(\sqrt{\varepsilon} z^{\prime}, \frac{z^{\prime}}{\sqrt{\varepsilon}}\right) \tilde{v}_{\varepsilon}, \quad z^{\prime} \in \frac{\Omega-\xi(t)}{\sqrt{\varepsilon}},
\end{aligned}
$$


with

$$
\begin{gathered}
a_{\varepsilon}^{i j}\left(t, z^{\prime}\right)=a^{i j}\left(\xi(t)+\sqrt{\varepsilon} z^{\prime}, \xi(t) / \varepsilon+z^{\prime} / \sqrt{\varepsilon}\right)+T^{i j}\left(\xi(t)+\sqrt{\varepsilon} z^{\prime}, \xi(t) / \varepsilon+z^{\prime} / \sqrt{\varepsilon}\right), \\
h_{\varepsilon}^{j}\left(t, z^{\prime}\right)=\left(\bar{b}^{j}\left(\sqrt{\varepsilon} z^{\prime}+\xi(t)\right)-\bar{b}^{j}(\xi(t))\right) / \sqrt{\varepsilon}+\sqrt{\varepsilon} \tilde{q}_{\xi, \frac{\xi}{\varepsilon}}^{j}\left(\sqrt{\varepsilon} z^{\prime}, \frac{z^{\prime}}{\sqrt{\varepsilon}}\right)=z_{i}^{\prime} \frac{\partial \bar{b}^{j}}{\partial x_{i}}(\xi(t))+o(1), \\
\rho_{\varepsilon}\left(t, z^{\prime}\right)=\frac{1}{\varepsilon} \rho\left(\xi(t)+\sqrt{\varepsilon} z^{\prime}\right)=\varrho^{2}\left(t, z^{\prime}\right)+o(1) ;
\end{gathered}
$$

here $\varrho\left(t, z^{\prime}\right)$ is the distance from $z^{\prime}$ to the line $\ell(t)=\left\{z^{\prime}=\alpha \bar{b}(\xi(t)) ; \alpha \in \mathbb{R}\right\}$, and $o(1)$ stands for a function that tends to zero uniformly in $t \in[0, P)$ and $z^{\prime}$ on every compact $K$.

Lemma 2.1. The first eigenvalues $\lambda_{\varepsilon}$ satisfies the estimate

$$
-C \leq \lambda_{\varepsilon} \leq \max \{c(x, y) ; x \in \bar{\Omega}, y \in \bar{Y}\}
$$

with a constant $C>0$ that does not depend on $\varepsilon$.

Proof. The upper bound for $\lambda_{\varepsilon}$ easily follows from the maximum principle.

To derive a lower bound for $\tilde{\lambda}_{\varepsilon}$ (and therefore $\lambda_{\varepsilon}$ ) we make use of the Aronson estimates [6]. Note that the coefficients in (2.11) are uniformly bounded, the principal term is uniformly elliptic, and for all sufficiently small $\varepsilon$ the domain $(\Omega-\xi(t)) / \sqrt{\varepsilon}$ contains the ball $B_{2}=\left\{z^{\prime} ;\left|z^{\prime}\right|<2\right\}$. We introduce an auxiliary function $g_{\varepsilon}\left(t, z^{\prime}\right)$ as a solution to the following parabolic problem,

$$
\begin{gathered}
-\frac{\partial g_{\varepsilon}}{\partial t}+\frac{\partial}{\partial z_{i}^{\prime}}\left(a_{\varepsilon}^{i j}\left(t, z^{\prime}\right) \frac{\partial g_{\varepsilon}}{\partial z_{j}^{\prime}}\right)+h_{\varepsilon}^{j}\left(t, z^{\prime}\right) \frac{\partial g_{\varepsilon}}{\partial z_{j}^{\prime}}+\left(c_{\xi, \frac{\xi}{\varepsilon}}\left(\sqrt{\varepsilon} z^{\prime}, \frac{z^{\prime}}{\sqrt{\varepsilon}}\right)-\delta \rho_{\varepsilon}\left(t, z^{\prime}\right)\right) g_{\varepsilon}=0 \quad \text { in }(0,+\infty) \times B_{2}, \\
g_{\varepsilon}\left(0, z^{\prime}\right)=\tilde{v}_{\varepsilon}\left(0, z^{\prime}\right), \quad g_{\varepsilon}=0 \quad \text { on }(0,+\infty) \times \partial B_{2} .
\end{gathered}
$$

Then

$$
f_{\varepsilon}:=g_{\varepsilon}\left(t, z^{\prime}\right)-\exp \left(t m_{\varepsilon}^{*} \tilde{\lambda}_{\varepsilon}\right) \tilde{v}_{\varepsilon}\left(t, z^{\prime}\right) \leq 0,
$$

where $m_{\varepsilon}^{*}:=\min \left\{\theta^{*}(x, y) ; x \in \bar{\Omega}, u \in \bar{Y}\right\}$ if $\tilde{\lambda}_{\varepsilon}<0$ and $m_{\varepsilon}^{*}:=\max \left\{\theta^{*}(x, y) ; x \in \bar{\Omega}, u \in \bar{Y}\right\}$ if $\tilde{\lambda}_{\varepsilon} \geq 0$. Indeed, $f_{\varepsilon}\left(0, z^{\prime}\right)=0$ in $B_{2}$ and $f_{\varepsilon}\left(t, z^{\prime}\right)<0$ on $\partial B_{2}$ for $t>0$, while

$$
\frac{\partial f_{\varepsilon}}{\partial t}-\frac{\partial}{\partial z_{i}^{\prime}}\left(a_{\varepsilon}^{i j}\left(t, z^{\prime}\right) \frac{\partial f_{\varepsilon}}{\partial z_{j}^{\prime}}\right)-h_{\varepsilon}^{j}\left(t, z^{\prime}\right) \frac{\partial f_{\varepsilon}}{\partial z_{j}^{\prime}}+\left(\delta \rho_{\varepsilon}\left(t, z^{\prime}\right)-c_{\xi, \frac{\xi}{\varepsilon}}\left(\sqrt{\varepsilon} z^{\prime}, \frac{z^{\prime}}{\sqrt{\varepsilon}}\right)\right) f_{\varepsilon} \leq 0 \quad \text { in }(0,+\infty) \times B_{2} .
$$

It follows by the maximum principle that $f_{\varepsilon} \leq 0$. On the other hand, applying the Aronson estimates to (2.12), we conclude that

$$
\min \left\{g_{\varepsilon}\left(P, z^{\prime}\right) ;\left|z^{\prime}\right| \leq 1\right\} \geq M^{\prime} \min \left\{g_{\varepsilon}\left(0, z^{\prime}\right) ;\left|z^{\prime}\right| \leq 1\right\}
$$

with $M^{\prime}>0$ independent of $\varepsilon$. Thus, due to the $P$-periodicity of $v_{\varepsilon}\left(t, z^{\prime}\right)$ in $t$ we have $\exp \left(P m_{\varepsilon}^{*} \tilde{\lambda}_{\varepsilon}\right) \geq M^{\prime}$ or $\tilde{\lambda}_{\varepsilon} \geq \log M^{\prime} /\left(P m_{\varepsilon}^{*}\right) \geq-C$, where $C>0$ is some constant independent of $\varepsilon$.

From Lemma 2.1 we conclude that $\tilde{\lambda}_{\varepsilon} \rightarrow \tilde{\lambda}$, up to extracting a subsequence. By parabolic estimates we then have, possibly passing to a further subsequence, $\tilde{v}_{\varepsilon} \rightarrow \tilde{v}$ in $C([0, P] \times K)$ and weakly in $L^{2}\left((0, P) ; H^{1}(K)\right)$ for every compact $K$; the limit function $\tilde{v}$ being positive.

Lemma 2.2. The function $\tilde{v}$ is a positive solution of

$$
-\frac{\partial \tilde{v}}{\partial t}+\frac{\partial}{\partial z_{i}^{\prime}}\left(\tilde{Q}^{i j}(\xi(t)) \frac{\partial \tilde{v}}{\partial z_{j}^{\prime}}\right)+z_{i}^{\prime} \frac{\partial \bar{b}^{j}}{\partial x_{i}}(\xi(t)) \frac{\partial \tilde{v}}{\partial z_{j}^{\prime}}+\left(\bar{c}(\xi(t))-\delta \varrho^{2}\left(t, z^{\prime}\right)\right) \tilde{v}=\tilde{\lambda} \tilde{v}, \quad z^{\prime} \in \mathbb{R}^{N},
$$


where the coefficients $\tilde{Q}^{i j}(\xi(t))$ are continuous P-periodic functions, the matrix $\tilde{Q}(\xi(t))=\left(\tilde{Q}^{i j}(\xi(t))\right)_{i, j=\overline{1, N}}$ is symmetric and satisfies the uniform ellipticity condition, and

$$
\bar{c}(\xi)=\int_{Y} c(\xi, y) \mathrm{d} y .
$$

Furthermore, $\tilde{v}$ inherits the P-periodicity in $t$, and satisfies the equation

$$
\bar{b}^{j}(\xi(t)) \frac{\partial \tilde{v}}{\partial z_{j}^{\prime}}=0 \quad \text { for all } t \in \mathbb{R} \text { and } z^{\prime} \in \mathbb{R}^{N} .
$$

Proof. The fact that $(\tilde{\lambda}, \tilde{v})$ satisfies $(2.13)$ can be justified by means of the standard homogenization techniques based on the div-curl lemma. The $P$-periodicity of $\tilde{v}\left(t, z^{\prime}\right)$ in $t$ is obvious.

To obtain relation (2.14) we get from (2.9)

$$
\sqrt{\varepsilon} \frac{\partial \tilde{v}_{\varepsilon}}{\partial t}=-\bar{b}^{j}(\xi(t)) \frac{\partial \tilde{v}_{\varepsilon}}{\partial z_{j}^{\prime}}
$$

which yields $\bar{b}^{j}(\xi(t)) \frac{\partial \tilde{v}_{\varepsilon}}{\partial z_{j}^{\prime}} \rightarrow 0$ in the sense of distributions. Since $\tilde{v}$ is $C^{1}$-smooth, being a solution of (2.13), we obtain the desired relation (2.14).

Relation (2.14) shows that the function $\tilde{v}$ does not depend on the variable directed along the vector field $\bar{b}(\xi(t))$. Thus, it is natural to eliminate this variable. To this end we introduce Cartesian coordinates $z_{1}, \ldots, z_{N-1}, \zeta$ so that $z_{1}, \ldots, z_{N-1}$ represent coordinates in the hyperplane orthogonal to the tangent line to $\mathcal{C}$ at $\xi(t)$. These coordinates can be chosen so that $(z, \zeta)=\mathcal{T}(t) z^{\prime}$ with the transformation matrix $\mathcal{T}(t)$ being $C^{2}$-smooth in $t$. Then by $(2.14), \tilde{v}\left(t, z^{\prime}\right)$ can be represented as a function $\tilde{V}(t, z)$ in coordinates $z_{1}, \ldots, z_{N-1}$ and (2.13) rewrites as

$$
-\frac{\partial \tilde{V}}{\partial t}+Q^{i j}(t) \frac{\partial^{2} \tilde{V}}{\partial z_{i} \partial z_{j}}+z_{i} B^{j i}(t) \frac{\partial \tilde{V}}{\partial z_{j}}+\left(\bar{c}(\xi(t))-\delta|z|^{2}\right) \tilde{V}=\tilde{\lambda} \tilde{V}, \quad z \in \mathbb{R}^{N-1}
$$

where

$$
B^{j i}(t)=\mathcal{T}^{i l}(t) \frac{\partial \bar{b}^{k}}{\partial x_{l}}(\xi(t)) \mathcal{T}^{j k}(t)-\dot{\mathcal{T}^{i l}}(t) \mathcal{T}^{j l}(t)
$$

All the coefficients in (2.15) as well as the function $\tilde{V}$ are $P$-periodic in $t$, and $Q^{i j}$ enjoy the symmetry $Q^{i j}=Q^{j i}$ and satisfy the uniform ellipticity condition.

Remark 2.3. If one linearizes the ODE $\dot{x}=-\bar{b}(x)$ near $\mathcal{C}$ to find $\dot{z}_{i}^{\prime}=-\frac{\partial \bar{b}^{i}}{\partial x_{j}}(\xi(t)) z_{j}^{\prime}$ and then applies the transformation $(z, \zeta)=\mathcal{T}(t) z^{\prime}$, then the resulting ODE is $\dot{z}=-B(t) z$.

Finally note that $\tilde{V}>0$ and $\tilde{V} \leq C$. This latter property follows from the uniform boundedness of the first eigenfunction $\tilde{u}_{\varepsilon}$ of (2.6). Let us prove this uniform boundedness. If $\tilde{u}_{\varepsilon}$ attains a (global) maximum at $x_{\varepsilon}$ then $\rho\left(x_{\varepsilon}\right) \leq \varepsilon\left(-\tilde{\lambda}_{\varepsilon}+c\left(x_{\varepsilon}, \frac{x_{\varepsilon}}{\varepsilon}\right)\right) / \delta$ and using the uniform lower bound for $\tilde{\lambda}_{\varepsilon}$ we conclude that $\operatorname{dist}\left(x_{\varepsilon}, \mathcal{C}\right) \leq C \sqrt{\varepsilon}$. On the other hand, the functions $\tilde{u}_{\varepsilon}\left(\xi+\sqrt{\varepsilon} z^{\prime}\right)$ are bounded on compacts, uniformly in $\xi \in \mathcal{C}$ and $\varepsilon$, therefore $\tilde{v} \leq C$. Thus $\tilde{V}$ is a bounded positive solution of (2.15). This fact will allow us to identify uniquely $\tilde{\lambda}$ and $\tilde{V}$ (up to normalization) in Section 3.2. 


\subsection{Blow up limits of eigenfunctions}

In this subsection we turn to equation (2.1). If we normalize its eigenfunctions $u_{\varepsilon}$ by $u_{\varepsilon}\left(\xi_{0}\right)=1$, the arguments from the previous subsection show that, up to extracting a subsequence, functions $w_{\varepsilon}(t, z, \zeta):=u_{\varepsilon}(\xi(t)+$ $\left.\sqrt{\varepsilon}(\mathcal{T}(t))^{-1}(z, \zeta)\right)$ converge (locally) uniformly to a positive solution $w(t, z)$ of

$$
-\frac{\partial w}{\partial t}+Q^{i j}(t) \frac{\partial^{2} w}{\partial z_{i} \partial z_{j}}+z_{i} B^{j i}(t) \frac{\partial w}{\partial z_{j}}+\bar{c}(\xi(t)) w=\lambda w, \quad z \in \mathbb{R}^{N-1} .
$$

Here $\lambda$ stands for a partial limit of eigenvalues $\lambda_{\varepsilon}$. However this time $w(t, z)$ could be an unbounded solution. In order to identify $\lambda$ and $w$ we need to know the asymptotic behavior of $w(t, z)$ as $|z| \rightarrow \infty$. This issue will be addressed in Section 4.

\section{Parabolic eigenvalue problems, existence and uniqueness}

In this section we study eigenvalue problems (2.15) and (2.17) in the class of positive $P$-periodic in $t$ functions. We begin with constructing solutions of a special form.

\subsection{Construction of eigenpairs via solutions of Riccati matrix equations}

Let us seek for a solution $\tilde{V}$ of (2.15) in the form $\tilde{V}(t, z)=\exp \left(-\Gamma_{\delta}^{i j}(t) z_{i} z_{j}+\phi_{\delta}(t)\right)$. This leads to the Riccati matrix equation

$$
-\dot{\Gamma}_{\delta}=4 \Gamma_{\delta} Q(t) \Gamma_{\delta}-\Gamma_{\delta} B(t)-B^{*}(t) \Gamma_{\delta}-\delta I,
$$

and the ODE

$$
-\dot{\phi}_{\delta}-2 \operatorname{tr}\left(Q(t) \Gamma_{\delta}(t)\right)+\bar{c}(\xi(t))=\tilde{\lambda} .
$$

We seek for a symmetric positive definite $\Gamma_{\delta}$, and both $\Gamma_{\delta}$ and $\phi_{\delta}$ must be $P$-periodic.

Since $Q(t)$ is positive definite, it is known [1], Theorem 5.3.4 that there exists a maximal symmetric $P$-periodic solution $\Gamma_{\delta}$ of (3.1). Its maximality is understood in the sense of quadratic forms, moreover $\Gamma_{\delta} \geq \Gamma$ for every symmetric $P$-periodic solution of the differential inequality $-\dot{\Gamma}-\mathcal{R}_{\delta}(t, \Gamma) \geq 0$, where $\mathcal{R}_{\delta}(t, \Gamma)$ stands for the Riccati map defined by the right hand side of (3.1). (To be consistent with the literature one should reverse the time in (3.1)). In order to prove that $\Gamma_{\delta}>0$ for $\delta>0$ one can take $\Gamma=r I$ and observe that $-\dot{\Gamma}-\mathcal{R}_{\delta}(t, \Gamma) \geq 0$ for sufficiently small $r>0$, this yields $\Gamma_{\delta} \geq r I$. Having defined $\Gamma_{\delta}$, we find the unique

$$
\tilde{\lambda}=-\frac{2}{P} \int_{0}^{P} \operatorname{tr}\left(Q(t) \Gamma_{\delta}(t)\right) \mathrm{d} t+\frac{1}{P} \int_{0}^{P} \bar{c}(\xi(t)) \mathrm{d} t
$$

such that (3.2) has a $P$-periodic solution $\phi_{\delta}(t)$.

In what concerns $(2.17)$, it has a solution $w(t, z)=\exp \left(-\Gamma_{0}^{i j}(t) z_{i} z_{j}+\phi_{0}(t)\right)$ with $\Gamma_{0}$ and $\phi_{0}$ solving

$$
-\dot{\Gamma}_{0}-\mathcal{R}_{0}\left(t, \Gamma_{0}\right)=0, \quad-\dot{\phi}_{0}-2 \operatorname{tr}\left(Q(t) \Gamma_{0}(t)\right)+\bar{c}(\xi(t))=\lambda .
$$

As before, $\Gamma_{0}$ is the maximal symmetric $P$-periodic solution of the Riccati equation in (3.4) whose existence is granted by Theorem 5.3.4 of [1]. Note that $\Gamma_{0}$ need not to be positive definite but rather positive semi definite.

\subsection{Uniqueness of eigenvalues and eigenfunctions}

In this subsection we prove a uniqueness result for problems (2.15) and (2.17). To define the class within which (2.17) is uniquely solvable we introduce special $P$-periodic solutions of the Lyapunov matrix equations

$$
-\dot{A}_{s}+A_{s} B(t)+B^{*}(t) A_{s}=\Pi_{s}^{*}(t) \Pi_{s}(t), \quad-\dot{A}_{u}+A_{u} B(t)+B^{*}(t) A_{u}=-\Pi_{u}^{*}(t) \Pi_{u}(t),
$$


here $B$ being defined in (2.16) and the right hand sides being chosen as follows. Consider the fundamental matrix solution $F(t, \tau)$ of

$$
\frac{\partial}{\partial t} F(t, \tau)=-B(t) F(t, \tau), \quad F(\tau, \tau)=I .
$$

It is well-known that $F(t+P, t)$ is $P$-periodic and its eigenvalues, called characteristic multipliers, are independent of $t$. Let $\Pi_{s}(t)$ and $\Pi_{u}(t)$ be the spectral projectors on the invariant subspaces of $F(t+P, t)$ corresponding to eigenvalues with absolute values strictly less than 1 and strictly greater than 1 . Then one checks that $A_{s}$ and $A_{u}$ given by

$$
A_{s}(t)=\int_{t}^{+\infty} F^{*}(\tau, t) \Pi_{s}^{*}(\tau) \Pi_{s}(\tau) F(\tau, t) \mathrm{d} \tau \quad \text { and } \quad A_{u}(t)=\int_{-\infty}^{t} F^{*}(\tau, t) \Pi_{u}^{*}(\tau) \Pi_{u}(\tau) F(\tau, t) \mathrm{d} \tau
$$

are $P$-periodic solutions of equation (3.5).

\section{Lemma 3.1.}

(i) There is a unique $\tilde{\lambda}$ such that (2.15) has a positive, bounded P-periodic in $t$ solution $\tilde{V}$, and this solution is unique up to multiplication by a positive constant.

(ii) Eigenvalue problem (2.17) is uniquely solvable in the class of positive functions $w$, P-periodic in $t$, and satisfying

$$
|w(t, z)| \exp \left(\mu A_{s}^{i j}(t) z_{i} z_{j}-\nu A_{u}^{i j}(t) z_{i} z_{j}\right) \text { is bounded for some } \mu>0 \text { and every } \nu>0 .
$$

That is $\lambda$ is unique and $w$ is unique up to multiplication by a positive constant.

Proof. The uniqueness result is justified by reducing (2.15) or (2.17) to the equation of the form

$$
-\frac{\partial u}{\partial t}+Q^{i j}(t) \frac{\partial^{2} u}{\partial z_{i} \partial z_{j}}+z_{i} \tilde{B}^{j i}(t) \frac{\partial u}{\partial z_{j}}+\tilde{C}(t, z) u=\sigma u, \quad z \in \mathbb{R}^{N-1}, t \in \mathbb{R}
$$

for a positive $P$-periodic in $t$ function $u(t, z)$ vanishing as $|z| \rightarrow \infty$. If $\tilde{C}(t, z) \rightarrow-\infty$ as $|z| \rightarrow \infty$, the latter problem has a unique solution according to Lemma A.1 (see the Appendix).

For (i) we use the transformation $u(t, z)=\mathrm{e}^{-r|z|^{2}} \tilde{V}(t, z)$ with $r>0$, this leads to the equation of the form (3.9) with $\tilde{C}(t, z)=4 r^{2} Q^{i j}(t) z_{i} z_{j}-2 r\left(B^{i j}(t) z_{i} z_{j}+\operatorname{tr} Q(t)\right)+\bar{c}(\xi(t))-\delta|z|^{2}$ and $\sigma=\tilde{\lambda}$. If we chose $r>0$ sufficiently small, we have $\tilde{C}(t, z) \rightarrow-\infty$ as $|z| \rightarrow \infty$.

For (ii) we set $\phi_{r}(t, z)=r A_{s}^{i j}(t) z_{i} z_{j}-r A_{u}^{i j}(t) z_{i} z_{j}$, where $A_{s}$ and $A_{u}$ are given by (3.7), $r>0$, and use the transformation $u(t, z)=\mathrm{e}^{\phi_{r}(t, z)} w(t, z)$. Then $u$ satisfies (3.9) with

$\tilde{C}=\bar{c}(\xi(t))+4 r^{2}\left(A_{u}^{i l}-A_{s}^{i l}\right) Q^{l m}\left(A_{u}^{m j}-A_{s}^{m j}\right) z_{i} z_{j}+r\left(\left(-\dot{A}_{u}^{i j}+\dot{A}_{s}^{i j}+2 B^{l i}\left(A_{u}^{l j}-A_{s}^{l j}\right)\right) z_{i} z_{j}+2 \operatorname{tr}\left(Q\left(A_{u}-A_{s}\right)\right)\right)$.

Since $\left.-\dot{A}_{u}^{i j}+\dot{A}_{s}^{i j}+2 B^{l i}\left(A_{u}^{l j}-A_{s}^{l j}\right)\right) z_{i} z_{j}=-\left|\Pi_{u} z\right|^{2}-\left|\Pi_{s} z\right|^{2}$ (by (3.5)), we have $\tilde{C}(t, z) \rightarrow-\infty$ as $|z| \rightarrow \infty$, provided that $r>0$ is sufficiently small. It is also clear from (3.8) that $u(t, z) \rightarrow 0$ as $|z| \rightarrow \infty$, when $0<r<\mu$.

To complete the proof it remains to show that solutions of (2.15) and (2.17) constructed in Section 3.1 do satisfy conditions of the lemma. To this end we prove the inequalities $\Gamma_{\delta} \geq 0$ and $\Gamma_{0} \geq \mu A_{s}$ for some $\mu>0$, where $\Gamma_{\delta}$ and $\Gamma_{0}$ are maximal solutions of the Riccati equations $-\dot{\Gamma}_{\delta}-\mathcal{R}_{\delta}\left(t, \Gamma_{\delta}\right)=0$ and $-\dot{\Gamma}_{0}-\mathcal{R}_{0}\left(t, \Gamma_{0}\right)=0$. The first inequality is already obtained in Section 3.1, even the strict one. To verify the second inequality we show that $-\mu \dot{A}_{s}-\mathcal{R}_{0}\left(t, \mu A_{s}\right) \geq 0$ for sufficiently small $\mu>0$. Since $A_{s}$ solves the first equation in (3.5), we have the equality $-\mu \dot{A}_{s}-\mathcal{R}_{0}\left(t, \mu A_{s}\right)=-4 \mu^{2} A_{s} Q A_{s}+\mu \Pi_{s}^{*} \Pi_{s}$ whose right hand side can be made non-negative by choosing small $\mu>0$, provided that $A_{s}(t) \leq C \Pi_{s}^{*}(t) \Pi_{s}(t)$ holds with some $C$ independent of $t$. On the other hand we know that $F(\tau+P, \tau)=F(\tau, t) F(t+P, t) F^{-1}(\tau, t)$ and therefore $\Pi_{s}(\tau) F(\tau, t)=F(\tau, t) \Pi_{s}(t)$. Thus $A_{s}$ can be represented as

$$
A_{s}(t)=\int_{t}^{+\infty} \Pi_{s}^{*}(t) F^{*}(\tau, t) F(\tau, t) \Pi_{s}(t) \mathrm{d} \tau
$$

hence the inequality $A_{s}(t) \leq C \Pi_{s}^{*}(t) \Pi_{s}(t)$ holds true with some $C$ independent of $t$. 
Remark 3.2. Condition (3.8) can be equivalently reformulated as

$$
|w(t, z)| \exp \left(\mu^{\prime} \Pi_{s}^{i j}(t) z_{i} z_{j}-\nu^{\prime} \Pi_{u}^{i j}(t) z_{i} z_{j}\right) \text { is bounded for some } \mu^{\prime}>0 \text { and every } \nu^{\prime}>0 .
$$

This is due to representation (3.10) for $A_{s}$ and similar representation for $A_{u}$ that reads

$$
A_{u}(t)=\int_{-\infty}^{t} \Pi_{u}^{*}(t) F^{*}(\tau, t) F(\tau, t) \Pi_{u}(t) \mathrm{d} \tau .
$$

\section{Limits OF EIGENVALUES AND SELECTION OF ADDITIVE EIGENFUNCTION}

We know (Sect. 2.1) that eigenvalues $\lambda_{\varepsilon}$ have the following bound

$$
\liminf \lambda_{\varepsilon} \geq-\frac{2}{P} \int_{0}^{P} \operatorname{tr}\left(Q \Gamma_{\delta}\right) \mathrm{d} t+\frac{1}{P} \int_{0}^{P} \bar{c}(\xi(t)) \mathrm{d} t
$$

for every $\delta>0$, where $\Gamma_{\delta}$ is the maximal $P$-periodic solution of (3.1). Since $-\dot{\Gamma}_{\delta^{\prime}}-\mathcal{R}_{\delta}\left(t, \Gamma_{\delta^{\prime}}\right)=-\dot{\Gamma}_{\delta^{\prime}}-$ $\mathcal{R}_{\delta^{\prime}}\left(t, \Gamma_{\delta^{\prime}}\right)+\left(\delta-\delta^{\prime}\right) I \geq 0$ for $\delta \geq \delta^{\prime}$, we have $\Gamma_{\delta} \geq \Gamma_{\delta^{\prime}}$. It follows that $\Gamma_{\delta}$ converges to the maximal $P$-periodic solution $\Gamma_{0}$ of $-\dot{\Gamma}_{0}-\mathcal{R}_{0}\left(t, \Gamma_{0}\right)=0$ as $\delta \rightarrow 0$, thus

$$
\liminf \lambda_{\varepsilon} \geq-\frac{2}{P} \int_{0}^{P} \operatorname{tr}\left(Q \Gamma_{0}\right) \mathrm{d} t+\frac{1}{P} \int_{0}^{P} \bar{c}(\xi(t)) \mathrm{d} t .
$$

The matching upper bound will be shown to hold in the case when $\mathcal{C}$ is a minimal component of the Aubry set $\mathcal{A}_{\bar{H}}$ with respect to a partial order relation similar to that defined in [13]. For reader's convenience we recall below necessary definitions from [13].

\subsection{Order relation on the Aubry set}

We recall that $W_{\varepsilon}=-\varepsilon \log u_{\varepsilon}$. It is shown in [13] that, up to a subsequence, $W_{\varepsilon} \rightarrow W$ in $C_{\text {loc }}(\Omega)$ and every limit function $W$ satisfies

$$
\bar{H}(\nabla W(x), x)=0 \text { in } \Omega, \quad \bar{H}(\nabla W(x), x) \geq 0 \text { on } \partial \Omega .
$$

Let us fix a converging subsequence $W_{\varepsilon}$. The limit function $W$ being a solution of (4.3) has the representation (see e.g., $[15,16])$

$$
W(x)=\min \left\{d_{\bar{H}}(x, y)+W(y) ; y \in \mathcal{A}_{\bar{H}}\right\},
$$

where $d_{\bar{H}}(x, y)$ is the distance function given by

$$
d_{\bar{H}}(x, y)=\sup \{V(x)-V(y) ; V \in C(\bar{\Omega}) \text { satisfies } \bar{H}(\nabla V(x), x) \leq 0 \text { in } \Omega\} .
$$

According to the assumption (1.9) the set $\mathcal{A}_{\bar{H}}$ can be divided into a finite number of connected components (fixed points or limit cycles of the ODE $\dot{x}=-\bar{b}(x)$ ), and we introduce the (partial) order relation $\preceq$ on the components of $\mathcal{A}_{\bar{H}}$ by setting

$$
\mathcal{A}^{\prime} \preceq \mathcal{A} \Longleftrightarrow d_{\bar{H}}\left(\mathcal{A}, \mathcal{A}^{\prime}\right)=W(\mathcal{A})-W\left(\mathcal{A}^{\prime}\right)
$$

(recall that $W$ is a constant on every component of the Aubry set). Choosing a minimal element(component) $\mathcal{A}$ we have (see details in [13])

$$
W(x)=d_{\bar{H}}(x, \mathcal{A})+W(\mathcal{A}) \quad \text { in a neighborhood of } \mathcal{A} .
$$




\subsection{Upper bound for eigenvalues}

The following statement completes the proof of the assertion (i) in Theorem 1.1. In this statement we deal with the case of a limit cycle of the equation $\dot{x}=-\bar{b}(x)$. The case when the minimal component of $\mathcal{A}_{\bar{H}}$ is a fixed point, say $\xi$, was considered in [13] under the assumption that $c=0$ in (1.1). If $c \neq 0$, then, following the line of [13], one can show that

$$
\lim \lambda_{\varepsilon}=\left(\sigma_{1}(\xi)+\sigma_{2}(\xi)\right)
$$

with $\sigma_{1}(\xi)$ and $\sigma_{2}(\xi)$ defined in Theorem 1.1. We leave the details to the reader.

Lemma 4.1. Let the limit cycle $\mathcal{C}$ be a minimal component of $\mathcal{A}_{\bar{H}}$, then

$$
\lim \lambda_{\varepsilon}=-\frac{2}{P} \int_{0}^{P} \operatorname{tr}\left(Q \Gamma_{0}\right) \mathrm{d} t+\frac{1}{P} \int_{0}^{P} \bar{c}(\xi(t)) \mathrm{d} t,
$$

where $\Gamma_{0}$ is the maximal P-periodic solution of $-\dot{\Gamma}_{0}-\mathcal{R}_{0}\left(t, \Gamma_{0}\right)=0$.

Proof. We begin with constructing a local subsolution $\Phi_{\mu}^{\nu}(x)$ of

$$
\bar{H}\left(\nabla \Phi_{\mu}^{\nu}(x), x\right) \leq-\delta \operatorname{dist}^{2}(x, \mathcal{C}) \quad \text { in a neighborhood of } \mathcal{C},
$$

where $\delta>0$. Assuming for the moment that $\Phi_{\mu}^{\nu}(x)$ is constructed, we have

Lemma 4.2. If $\Phi_{\mu}^{\nu}$ satisfies (4.8) and $\Phi_{\mu}^{\nu}=W$ on $\mathcal{C}$ then the strict inequality $W>\Phi_{\mu}^{\nu}$ holds in $\overline{U_{\mathcal{C}}} \backslash \mathcal{C}$, where $U_{\mathcal{C}}$ is a neighborhood of $\mathcal{C}$.

Proof. The arguments follow those from [13] (Lem. 15), with minor modifications.

In constructing the function $\Phi_{\mu}^{\nu}$ we use the same trick as in Section 2, we pass to the moving coordinates $x^{\prime}=x-\xi(t)$ to find that (4.8) is equivalent to the following inequality for $\tilde{\Phi}_{\mu}^{\nu}\left(t, x^{\prime}\right):=\Phi_{\mu}^{\nu}\left(x^{\prime}+\xi(t)\right)$,

$$
\frac{\partial \tilde{\Phi}_{\mu}^{\nu}}{\partial t}+\bar{H}\left(\nabla_{x^{\prime}} \tilde{\Phi}_{\mu}^{\nu}, x^{\prime}+\xi(t)\right)+\bar{b}^{j}(\xi(t)) \frac{\partial \tilde{\Phi}_{\mu}^{\nu}}{\partial x_{j}^{\prime}} \leq-\delta \operatorname{dist}^{2}\left(x^{\prime}+\xi(t), \mathcal{C}\right)
$$

Linearizing $\bar{H}\left(p, x^{\prime}+\xi(t)\right)$ in $p$ and then in $x^{\prime}$,

$$
\bar{H}(p, x)=-\bar{b}^{j}(\xi(t)) p_{j}-x_{i}^{\prime} \frac{\partial \bar{b}^{j}}{\partial x_{i}}(\xi(t)) p_{j}+O\left(|p|^{2}+|p|\left|x^{\prime}\right|^{2}\right)
$$

and passing to the coordinates $(z, \zeta)=\mathcal{T}(t) x^{\prime}$ (where $z=\left(z_{1}, \ldots, z_{N-1}\right.$ ) are coordinates in the hyperplane orthogonal to the tangent line to $\mathcal{C}$ at $\xi(t), c f$. Sect. 2.1$)$, we construct $\Psi_{\mu}^{\nu}(t, z, \zeta)=\tilde{\Phi}_{\mu}^{\nu}\left(t, \mathcal{T}^{-1}(z, \zeta)\right)$ to satisfy

$$
\frac{\partial \Psi_{\mu}^{\nu}}{\partial t}-z_{i} B^{j i}(t) \frac{\partial \Psi_{\mu}^{\nu}}{\partial z_{j}} \leq-\delta^{\prime}|z|^{2} \quad\left(\delta^{\prime}>0\right), \quad \frac{\partial \Psi_{\mu}^{\nu}}{\partial \zeta}=0 .
$$

In addition, $\Psi_{\mu}^{\nu}(t, z, \zeta)$ should be $P$-periodic in $t$. All these requirements are fulfilled if we set

$$
\Psi_{\mu}^{\nu}(t, z, \zeta)=\Psi_{\mu}^{\nu}(t, z):=\mu A_{s}^{i j}(t) z_{i} z_{j}-\nu A_{u}^{i j}(t) z_{i} z_{j},
$$

$A_{s}$ and $A_{u}$ being solutions of Lyapunov matrix equations (3.5) given by (3.7). 
Lemma 4.3. The function

$$
\Phi_{\mu}^{\nu}(x)=\Psi_{\mu}^{\nu}\left(\xi^{-1}\left(X_{\mathcal{C}}(x)\right), \mathcal{T}\left(\xi^{-1}\left(X_{\mathcal{C}}(x)\right)\right)\left(x-X_{\mathcal{C}}(x)\right)\right),
$$

where $\Psi_{\mu}^{\nu}$ is given by (4.11), satisfies (4.8) for $0<\nu \leq \mu<r$, with $r>0$ sufficiently small $(\delta>0$ depends on $\mu, \nu)$. Here $X_{\mathcal{C}}(x)$ denotes the nearest point projection of $x$ on $\mathcal{C}, \xi^{-1}$ is the function inverse to $\xi:[0, P) \rightarrow \mathcal{C}$, $\xi(t)$ being the solution of (2.4).

Proof. To verify (4.8) we redo in details the above reasonings, which led us to (4.10). Namely, after changes of variables $(z, \zeta)=\mathcal{T}(t) x^{\prime}$ and unknown functions $\Psi_{\mu}^{\nu}(t, z, \zeta)=\tilde{\Phi}_{\mu}^{\nu}\left(t, \mathcal{T}^{-1}(z, \zeta)\right)$, we see that (4.8) leads to

$$
\frac{\partial \Psi_{\mu}^{\nu}}{\partial t}+z_{i} \dot{\mathcal{T}}^{j k}(t) \mathcal{T}^{i k}(t) \frac{\partial \Psi_{\mu}^{\nu}}{\partial z_{j}}+\bar{H}\left(\mathcal{T}(t) \nabla_{z} \Psi_{\mu}^{\nu}, \xi(t)+\mathcal{T}^{-1}(t)(z, 0)\right)+\bar{b}^{k}(\xi(t)) \mathcal{T}^{j k}(t) \frac{\partial \Psi_{\mu}^{\nu}}{\partial z_{j}} \leq-\delta|z|^{2} .
$$

Conversely, if this inequality holds for small $z$ uniformly in $t$, one readily checks that $\Phi_{\mu}^{\nu}(x)$, restored by the formula (4.12), does satisfy (4.8). Letting $J$ denote the left hand side of (4.13), we can bound it as

$$
\begin{aligned}
J= & \frac{\partial \Psi_{\mu}^{\nu}}{\partial t}+\left(\left(\bar{b}^{k}(\xi(t))-\bar{b}^{k}\left(\xi(t)+\mathcal{T}^{-1}(t)(z, 0)\right)\right) \mathcal{T}^{j k}(t)+z_{i} \dot{\mathcal{T}}^{j k}(t) \mathcal{T}^{i k}(t)\right) \frac{\partial \Psi_{\mu}^{\nu}}{\partial z_{j}}+I_{1} \\
& \leq \frac{\partial \Psi_{\mu}^{\nu}}{\partial t}+z_{i}\left(\dot{\mathcal{T}}^{j k}(t) \mathcal{T}^{i k}(t)-\mathcal{T}^{i m} \frac{\partial \bar{b}^{k}}{\partial x_{m}}(\xi(t)) \mathcal{T}^{j k}(t)\right) \frac{\partial \Psi_{\mu}^{\nu}}{\partial z_{j}}+I_{2},
\end{aligned}
$$

where $I_{1} \leq C\left|\nabla_{z} \Psi_{\mu}^{\nu}\right|^{2}, I_{2} \leq I_{1}+\bar{o}(|z|)\left|\nabla_{z} \Psi_{\mu}^{\nu}\right|$. Due to (3.10), (3.12) we have

$$
\left|\nabla_{z} \Psi_{\mu}^{\nu}\right|^{2} \leq C\left(\mu^{2}\left|\Pi_{s} z\right|^{2}+\nu^{2}\left|\Pi_{u} z\right|^{2}\right)
$$

and therefore

$$
I_{2} \leq C^{\prime}\left(\mu^{2}\left|\Pi_{s} z\right|^{2}+\nu^{2}\left|\Pi_{u} z\right|^{2}\right)+\bar{o}\left(|z|^{2}\right) .
$$

On the other hand, the middle term in the second line of (4.14) is nothing but $-z_{i} B^{j i}(t) \frac{\partial \Psi_{\mu}^{\nu}}{\partial z_{j}}$ and, since $A_{s}, A_{u}$ are solutions of the Lyapunov equations (3.5), the sum of all three terms equals $I_{2}-\mu\left|\Pi_{s} z\right|^{2}-\nu\left|\Pi_{u} z\right|^{2}$. Thus the statement of the lemma holds if $\mu, \nu<1 / C^{\prime}$, where $C^{\prime}$ is the constant appearing in the bound for $I_{2}$.

Remark 4.4. If we change the coordinates $(z, \eta)=\mathcal{T}(t)(x-\xi(t))$ then $\Phi_{\mu}^{\nu}\left((\mathcal{T}(t))^{-1}(z, 0)\right)=\Psi_{\mu}^{\nu}(t, z)$, where $\Psi_{\mu}^{\nu}(t, z)$ is given by (4.11). It follows that

$$
\Phi_{\mu}^{\nu}\left((\mathcal{T}(t))^{-1}(z, 0)\right) \geq \gamma_{1} \mu\left|\Pi_{s}(t) z\right|^{2}-\gamma_{2} \nu\left|\Pi_{u}(t) z\right|^{2}
$$

for some $\gamma_{1}, \gamma_{2}>0$.

Our next goal is to obtain exponential estimates for the function $w$ introduced in Section 2.2. Here we follow closely the line of [13]. We set $\bar{\Phi}_{\varepsilon}=\Phi_{\mu}^{\nu}(x)+\varepsilon \theta_{\varepsilon}\left(\nabla \tilde{\Phi}_{\varepsilon}(x), x, x / \varepsilon\right)$, where

$$
\tilde{\Phi}_{\varepsilon}(x)=\frac{1}{\varepsilon^{n}} \int_{\mathbb{R}^{N}} \varphi\left(\left(x-x^{\prime}\right) / \varepsilon\right) \Phi_{\mu}^{\nu}\left(x^{\prime}\right) \mathrm{d} x^{\prime}
$$

$\varphi$ being a smooth nonnegative function with compact support and $\int_{\mathbb{R}^{N}} \varphi(x) \mathrm{d} x=1$. Then by (4.8) and (1.4) we have, after routine computations,

$$
-\varepsilon a^{i j}(x, x / \varepsilon) \frac{\partial^{2} \bar{\Phi}_{\varepsilon}}{\partial x_{i} \partial x_{j}}+H\left(\nabla \bar{\Phi}_{\varepsilon}(x), x, x / \varepsilon\right) \leq-\delta \operatorname{dist}^{2}(x, \mathcal{C})+C \varepsilon \quad \text { in } U_{\mathcal{C}}
$$


By Lemma 4.2 the strict inequality $W_{\varepsilon}>\bar{\Phi}_{\varepsilon}$ holds on $\partial U_{\mathcal{C}}$ for small $\varepsilon$. Let us show that $W_{\varepsilon} \geq \bar{\Phi}_{\varepsilon}-\beta \varepsilon$ with $\beta$ independent of $\varepsilon$.

Assume that $W_{\varepsilon}-\bar{\Phi}_{\varepsilon}$ attains its negative minimum at $x_{\varepsilon}$, then $\nabla W_{\varepsilon}\left(x_{\varepsilon}\right)=\nabla \bar{\Phi}_{\varepsilon}\left(x_{\varepsilon}\right)$ and $a^{i j}\left(x_{\varepsilon}, x_{\varepsilon} / \varepsilon\right) \frac{\partial^{2} \bar{\Phi}_{\varepsilon}}{\partial x_{i} \partial x_{j}}\left(x_{\varepsilon}\right) \leq a^{i j}\left(x_{\varepsilon}, x_{\varepsilon} / \varepsilon\right) \frac{\partial^{2} W_{\varepsilon}}{\partial x_{i} \partial x_{j}}\left(x_{\varepsilon}\right)$. It follows by (1.2) and (4.15) that

$$
\begin{aligned}
\varepsilon \lambda_{\varepsilon}-\varepsilon c\left(x_{\varepsilon}, x_{\varepsilon} / \varepsilon\right)= & -\varepsilon a^{i j}\left(x_{\varepsilon}, x_{\varepsilon} / \varepsilon\right) \frac{\partial^{2} W_{\varepsilon}}{\partial x_{i} \partial x_{j}}\left(x_{\varepsilon}\right)+H\left(\nabla \bar{\Phi}_{\varepsilon}\left(x_{\varepsilon}\right), x_{\varepsilon}, x_{\varepsilon} / \varepsilon\right) \\
& \leq-\varepsilon a^{i j}\left(x_{\varepsilon}, x_{\varepsilon} / \varepsilon\right) \frac{\partial \bar{\Phi}_{\varepsilon}}{\partial x_{i} \partial x_{j}}\left(x_{\varepsilon}\right)+H\left(\nabla \bar{\Phi}_{\varepsilon}\left(x_{\varepsilon}\right), x_{\varepsilon}, x_{\varepsilon} / \varepsilon\right) \leq-\delta \operatorname{dist}^{2}\left(x_{\varepsilon}, \mathcal{C}\right)+C \varepsilon .
\end{aligned}
$$

Thus $\operatorname{dist}\left(x_{\varepsilon}, \mathcal{C}\right) \leq C \sqrt{\varepsilon}$ and $W_{\varepsilon} \geq \bar{\Phi}_{\varepsilon}+W_{\varepsilon}\left(x_{\varepsilon}\right)-\bar{\Phi}_{\varepsilon}\left(x_{\varepsilon}\right)$. Note that, since $\operatorname{dist}\left(x_{\varepsilon}, \mathcal{C}\right) \leq C \sqrt{\varepsilon}$, both $W_{\varepsilon}\left(x_{\varepsilon}\right) / \varepsilon$ and $\bar{\Phi}_{\varepsilon}\left(x_{\varepsilon}\right) / \varepsilon$ remain bounded in the limit $\varepsilon \rightarrow 0$ (the boundedness of $W_{\varepsilon}\left(x_{\varepsilon}\right) / \varepsilon$ follows from results of Section 2.1). Thus the limit $w$ of functions $w_{\varepsilon}(t, z, \zeta)=u_{\varepsilon}\left(\xi(t)+\sqrt{\varepsilon}(\mathcal{T}(t))^{-1}(z, \zeta)\right)$ satisfies

$$
|w(t, z)| \leq C \mathrm{e}^{-\liminf \bar{\Phi}_{\varepsilon}(\sqrt{\varepsilon} z) / \varepsilon} \leq C \mathrm{e}^{-\mu_{1}\left|\Pi_{s}(t) z\right|^{2}+\mu_{2}\left|\Pi_{u}(t) z\right|^{2}},
$$

where $\mu_{1}$ is a positive constant and $\mu_{2}$ can be chosen sufficiently small (constant $C$ in (4.16) depends on $\mu_{2}$ ). On the other hand $w$ solves (2.17). Thus, by Lemma 3.1 we have

$$
\lambda_{\varepsilon} \rightarrow-\frac{2}{P} \int_{0}^{P}\left(\operatorname{tr}\left(\Gamma_{0}(t) Q(t)\right) \mathrm{d} t+\frac{1}{P} \int_{0}^{P} \bar{c}(\xi(t)) \mathrm{d} t .\right.
$$

Lemma 4.1 is proved.

Now we can complete the

Proof of assertion (i) of Theorem 1.1. It follows from the analysis of [13] (up to minor modifications) that $\lim \inf \lambda_{\varepsilon} \geq \max \left\{\sigma_{1}(\xi)+\sigma_{2}(\xi)\right\}$, where the maximum is taken over all fixed points $\xi$ of $\dot{x}=-\bar{b}(x)$ if the set of fixed points is not empty. In Section 5 we prove that the first term in (4.2) coincides with $\sigma_{1}(\xi)$ given by (1.11) when $\xi$ is a point on a limit cycle (Prop. 5.1 below). Thus $\lim \inf \lambda_{\varepsilon} \geq \bar{\sigma}$, where $\bar{\sigma}$ is defined in (1.10). By (4.6) and (4.7) we then obtain (1.10).

\subsection{Selection of the additive eigenfunction}

We proceed with assertion (ii) of Theorem 1.1. We recall that due to the assumptions made in item (ii), the maximum in (1.10) is attained at exactly one component, we call this component $\mathcal{M}$. Then by Lemma 4.1 (when $\mathcal{M}$ is limit cycle) or (4.6) (when $\mathcal{M}$ is a fixed point), $\mathcal{M}$ is the unique minimal component of the Aubry set $\mathcal{A}_{\bar{H}}$. It follows that $\mathcal{M}$ is the least component in $\mathcal{A}_{\bar{H}}$ with respect to the order relation " $\preceq$ ". In other words we have

$$
W(\xi)=d_{\bar{H}}(\xi, \mathcal{M})+W(\mathcal{M}) \quad \forall \xi \in \mathcal{A}_{\bar{H}} .
$$

Then by (4.4) we obtain $W(x)=d_{\bar{H}}(x, \mathcal{M})+W(\mathcal{M})$.

\section{INVARIANT FORM OF EIGENVALUE ASYMPTOTICS VIA EIGENVALUES OF LINEARIZED POINCARÉ MAP}

The eigenvalue $\lambda$ of (2.17) is given by the formula $-\frac{2}{P} \int_{0}^{P} \operatorname{tr}\left(\Gamma_{0}(t) Q(t)\right) \mathrm{d} t+\frac{1}{P} \int_{0}^{P} \bar{c}(\xi(t)) \mathrm{d} t$, where $\Gamma_{0}(t)$ is the maximal $P$-periodic solution of the Riccati equation

$$
\dot{\Gamma}_{0}+4 \Gamma_{0} Q \Gamma_{0}-\Gamma_{0} B-B^{*} \Gamma_{0}=0 .
$$

Since $Q(t)$ and $B(t)$ depend on a particular choice of the transformation matrix $\mathcal{T}(t)$ (cf. Sect. 2.1), it is natural to express the eigenvalue in an invariant form. 
Proposition 5.1. Let $\Lambda_{k}$ be the eigenvalues of $F(\tau+P, \tau), F(t, \tau)$ being the fundamental matrix solution of (3.6). Assume that these eigenvalues $\Lambda_{k}$ does not lie on the unit circle. Then we have

$$
-\frac{2}{P} \int_{0}^{P} \operatorname{tr}\left(\Gamma_{0}(t) Q(t)\right) \mathrm{d} t=\frac{1}{P} \sum_{\left|\Lambda_{k}\right|<1} \log \left|\Lambda_{k}\right|
$$

where $\Gamma_{0}(t)$ is the maximal P-periodic solution of (5.1).

Proof. Rewrite (5.1) in the form

$$
\frac{\partial}{\partial t}\left(F^{*}(t, \tau) \Gamma_{0}(t) F(t, \tau)\right)+\left(4 F^{*}(t, \tau) \Gamma_{0}(t) Q(t)\left(F^{*}(t, \tau)\right)^{-1}\right) F^{*}(t, \tau) \Gamma_{0}(t) F(t, \tau)=0,
$$

multiplying (5.1) by $F^{*}(t, \tau)$ from the left and by $F(t, \tau)$ from the right, we have also used here (3.6).

Let us show first that

$$
\Gamma_{0}(\tau) \Pi_{u}(\tau)=\Pi_{u}^{*}(\tau) \Gamma_{0}(\tau)=0 \quad \text { and } \quad \Gamma_{0}(\tau) \Pi_{s}(\tau)=\Pi_{s}^{*}(\tau) \Gamma_{0}(\tau)=\Gamma_{0}(\tau),
$$

where $\Pi_{u}(\tau), \Pi_{s}(\tau)$ are projectors on the invariant subspaces of $F(\tau+P, \tau)$ corresponding to eigenvalues with moduli strictly grater than 1 and strictly less than 1 . To this end integrate (5.2) on $(\tau, \tau+P)$ and multiply the result by $\Pi_{u}^{*}(\tau)$ from the left and by $\Pi_{u}(\tau)$ from the right to get

$$
\Pi_{u}^{*}(\tau) F^{*}(\tau+P, \tau) \Gamma_{0}(\tau) F(\tau+P, \tau) \Pi_{u}(\tau)-\Pi_{u}^{*}(\tau) \Gamma_{0}(\tau) \Pi_{u}(\tau) \leq 0 .
$$

Since $\Pi_{u}(\tau)$ is a projector on the invariant subspace of $F(\tau+P, \tau)$ corresponding to eigenvalues with moduli strictly grater than 1 , we conclude that $\Gamma_{0}(\tau) \Pi_{u}(\tau)=0$. Consequently (5.3) does hold. Also, since $\Gamma_{0}(\tau)$ is the maximal solution of (5.1) we have $\Gamma_{0}(\tau) \geq \gamma \Pi_{s}^{*}(\tau) \Pi_{s}(\tau)$, with $\gamma>0$ (cf. proof of Lem. 3.1).

Let us pass to a new basis transforming $\Gamma_{0}$ into block diagonal form. Fix $\tau$ and consider an orthonormal frame $\left\{a_{1}, \ldots, a_{k}, \ldots a_{N-1}\right\}$ with the first $k$ vectors chosen to form a basis of $\operatorname{Im}\left(\Pi_{s}^{*}(\tau)\right)$. Rewriting matrices appearing in (5.2) in the aforementioned frame,

$$
\left(\Gamma^{\prime}(t)\right)^{i j}=a_{i} \cdot F^{*}(t, \tau) \Gamma_{0}(t) F(t, \tau) a_{j} \text { and }\left(M^{\prime}(t)\right)^{i j}=4 a_{i} \cdot F^{*}(t, \tau) \Gamma_{0}(t) Q(t)\left(F^{*}(t, \tau)\right)^{-1} a_{j},
$$

(5.2) reads

$$
\dot{\Gamma}^{\prime}(t)+M^{\prime}(t) \Gamma^{\prime}(t)=0
$$

Note that

$$
\left(\Gamma^{\prime}(t)\right)^{i j}=a_{i} \cdot F^{*}(t, \tau) \Pi_{s}^{*}(t) \Gamma_{0}(t) \Pi_{s}(t) F(t, \tau) a_{j}=a_{i} \cdot \Pi_{s}^{*}(\tau) F^{*}(t, \tau) \Gamma_{0}(t) F(t, \tau) \Pi_{s}(\tau) a_{j}=0
$$

if $i>k$ or $j>k$, also $\left(M^{\prime}(t)\right)^{i j}=0$ if $i>k$. Therefore

$$
4 \operatorname{tr}\left(\Gamma_{0}(t) Q(t)\right)=4 \operatorname{tr}\left(F^{*}(t, \tau) \Gamma_{0}(t) Q(t)\left(F^{*}(t, \tau)\right)^{-1}\right)=\sum_{1}^{k}\left(M^{\prime}(t)\right)^{i i} .
$$

Consider now matrices $\Gamma^{\prime \prime}(t)$ and $M^{\prime \prime}(t)$ corresponding to the upper $k \times k$ diagonal blocks of $\Gamma^{\prime}(t)$ and $M^{\prime}(t)$. We have $\dot{\Gamma}^{\prime \prime}(t)+M^{\prime \prime}(t) \Gamma^{\prime \prime}(t)=0$, moreover $\operatorname{tr}\left(\Gamma_{0}(t) Q(t)\right)=\frac{1}{4} \operatorname{tr} M^{\prime \prime}(t)$ and $\operatorname{det} \Gamma^{\prime \prime}(\tau) \neq 0\left(\Gamma^{\prime \prime}(\tau)>0\right.$ as follows from the bound $\Gamma_{0}(\tau) \geq \gamma \Pi_{s}^{*}(\tau) \Pi_{s}(\tau)$ with $\left.\gamma>0\right)$. Therefore by Liouville's formula

$$
\operatorname{det} \Gamma^{\prime \prime}(\tau+P) / \operatorname{det} \Gamma^{\prime \prime}(\tau)=\exp \left(-\int_{\tau}^{\tau+P} \operatorname{tr} M^{\prime \prime}(t) \mathrm{d} t\right)=\exp \left(-\int_{0}^{P} \operatorname{tr} M^{\prime \prime}(t) \mathrm{d} t\right) .
$$


Thus

$$
2 \int_{0}^{P} \operatorname{tr}\left(\Gamma_{0}(t) Q(t)\right) \mathrm{d} t=\frac{1}{2}\left(\log \operatorname{det} \Gamma^{\prime \prime}(\tau)-\log \operatorname{det} \Gamma^{\prime \prime}(\tau+P)\right) .
$$

It remains to calculate the right hand side of (5.4). To this end note that $\Gamma^{\prime \prime}(\tau+P)=\tilde{D}^{*} \Gamma^{\prime \prime}(\tau) \tilde{D}$, where $\tilde{D}=\left(a_{i} \cdot F(\tau+P, \tau) \Pi_{s}(\tau) a_{j}\right)_{i, j=\overline{1, k}}$. This latter relation follows from the equality $F^{*}(\tau+P, \tau) \Gamma_{0}(\tau+P) F(\tau+$ $P, \tau)=\Pi_{s}^{*}(\tau) F^{*}(\tau+P, \tau) \Gamma_{0}(\tau) F(\tau+P, \tau) \Pi_{s}(\tau)$ in conjunction with the fact that $\left(\Gamma^{\prime}(t)\right)^{i j}=0$ if $i>k$ or $j>k$. We now have

$$
2 \int_{0}^{P} \operatorname{tr}\left(\Gamma_{0}(t) Q(t)\right) \mathrm{d} t=-\log |\operatorname{det} \tilde{D}| .
$$

On the other hand, since vectors $\Pi_{s}(\tau) a_{1}, \ldots, \Pi_{s}(\tau) a_{k}$ form a basis of $\operatorname{Im}\left(\Pi_{s}(\tau)\right)$ and $a_{1}, \ldots, a_{k}$ are biorthogonal vectors, $\tilde{D}$ is nothing but the matrix of the restriction of $F(\tau+P, \tau)$ to $\operatorname{Im}\left(\Pi_{s}(\tau)\right)$. Consequently $|\operatorname{det} \tilde{D}|$ equals the product of absolute values strictly less than 1 of eigenvalues of $F(\tau+P, \tau)$.

In conclusion we note that according to Remark 2.3 the eigenvalues of $F(\tau+P, \tau)$ coincide with that of the linearized Poincaré map associated to the limit cycle $\mathcal{C}$.

\section{EXAMPLE}

Consider a particular case of equation (1.1) in 3D with the drift and the potential being independent of the fast variable. The equation takes the form

$$
\varepsilon a^{i j}\left(x, \frac{x}{\varepsilon}\right) \frac{\partial^{2} u}{\partial x_{i} \partial x_{j}}+b^{j}(x) \frac{\partial u}{\partial x_{j}}+c(x) u=\lambda u \quad \text { in } \Omega .
$$

In this case by (1.8) and (1.7) we have $\bar{b}(x)=b(x)$. Assume that the set of $\omega$-limit points of the ODE $\dot{x}=-b(x)$ in $\bar{\Omega}$ is nonempty, then the additive eigenvalue $\lambda_{\bar{H}}$ is zero and the Aubry set $\mathcal{A}_{\bar{H}}$ is completely determined by the drift $b(x)$, although the effective Hamiltonian $\bar{H}$ itself does depend on the coefficients $a^{i j}(x, y)$.

Let components of the drift $b(x)$ be given by

$$
\begin{aligned}
& b^{1}(x)=x_{2}+\left(x_{1}^{2}+x_{2}^{2}-1\right) x_{1}, \\
& b^{2}(x)=-x_{1}+\left(x_{1}^{2}+x_{2}^{2}-1\right) x_{2}, \\
& b^{3}(x)=\alpha x_{3},
\end{aligned}
$$

where $\alpha \neq 0$ is a parameter, and suppose that the domain $\Omega$ contains both the unit circle $S^{1}=\left\{x \in \mathbb{R}^{3}\right.$ : $\left.x_{1}^{2}+x_{2}^{2}=1, x_{3}=0\right\}$ on the $x_{1} x_{2}$ plain and zero point. Then we claim that $\mathcal{A}_{\bar{H}}=S^{1} \cup\{0\}$. To justify this we first observe that both $S^{1}$ and 0 belong to $\mathcal{A}_{\bar{H}}, S^{1}$ being the closed trajectory and 0 being a fixed point of the ODE $\dot{x}=-b(x)$. On the other hand a simple consideration of trajectories $x(t), t \geq 0$ of the ODE shows that if the initial value $x(0) \notin S^{1} \cup\{0\}$ then either $x(t)$ lefts $\Omega$ in the finite time or tends to $S^{1} \cup\{0\}$ as $t \rightarrow \infty$, additionally, there are exactly two full trajectories $x(t) \in \bar{\Omega},-\infty<t<\infty$ that are either point 0 or limit cycle $S^{1}$. This implies that indeed $\mathcal{A}_{\bar{H}}=S^{1} \cup\{0\}$.

In order to calculate the numbers $\sigma_{1}(\xi)$ and $\sigma_{2}(\xi)$ associated with the limit cycle $S^{1}\left(\xi \in S^{1}\right)$ via (1.11) and (1.12), it is useful to pass to the cylindric coordinates, $x_{1}=r \cos \varphi, x_{2}=r \sin \varphi, x_{3}=z$. The ODE $\dot{x}=-b(x)$ then reads

$$
\dot{r}=-\left(r^{2}-1\right) r, \quad \dot{\phi}=1, \quad \dot{z}=\alpha z .
$$

Thus the minimal period of the cycle is $P=2 \pi$, and linearizing the first equation and the last equation (6.1) around $r=1\left(r^{\prime}=r-1\right)$ and $z=0\left(z^{\prime}=z\right)$, we find the linearized Poincaré map: $r^{\prime} \mapsto \mathrm{e}^{-2 P} r^{\prime}, z^{\prime} \mapsto \mathrm{e}^{\alpha P} z^{\prime}$, whose eigenvalues are $\mathrm{e}^{-4 \pi}$ and $\mathrm{e}^{2 \alpha \pi}$. Now, due to (1.11) and (1.12), we have

$$
\sigma_{1}(\xi)=-2+\min \{\alpha, 0\}, \quad \sigma_{2}(\xi)=\frac{1}{2 \pi} \int_{S^{1}} c(x) \mathrm{d} s \quad \text { for } \xi \in S^{1} .
$$


In the case of the fixed point 0 , the eigenvalues of the matrix $\left(\frac{\partial b^{i}}{\partial x_{j}}(0)\right)_{i, j=\overline{1, N}}$ are $1 \pm \imath$ and $\alpha$, i.e. $\sigma_{1}(0)=$ $\min \{\alpha, 0\}$. Thus maximization problem (1.10) which determines the limit as $\varepsilon \rightarrow 0$ of the first eigenvalue in this example takes form

$$
\max \left\{-2+\min \{\alpha, 0\}+\frac{1}{2 \pi} \int_{S^{1}} c(x) \mathrm{d} s, \min \{\alpha, 0\}+c(0)\right\} .
$$

If the maximum in (6.2) is attained at the first number and not the second one, then the first eigenfunction concentrates on the circle $S^{1}$.

\section{Appendix A.}

The result presented in this Appendix generalizes a theorem in [18] about the existence and uniqueness of the principal eigenpair of elliptic operators to the case of periodic in time parabolic spectral problems. Namely, let $A_{t}$ be a $P$-periodic in $t$ family of elliptic operators in $\mathbb{R}^{N}$ of the form

$$
A_{t} u=a^{i j}(t) \frac{\partial^{2} u}{\partial x_{i} \partial x_{j}}+b(t, x) \cdot \nabla u+c(t, x) u
$$

We assume that the coefficients $a^{i j}(t)$ satisfy the ellipticity condition uniformly in $t$ and the symmetry $a^{i j}=a^{j i}$ holds; $|b(t, x)| \leq c_{1}+c_{2}|x| ; c(t, x)$ tends to $-\infty$ as $|x| \rightarrow \infty$ uniformly in $t$; all the coefficients are $C^{1}$ functions, $P$-periodic in $t$. We consider the spectral problem

$$
-\partial_{t} u+A_{t} u=\lambda u \quad \text { in } \mathbb{R} \times \mathbb{R}^{N}
$$

subject to the $P$-periodicity in $t$ condition and a decay condition as $|x| \rightarrow \infty$. We are interested in eigenfunctions that belong to the class $\Xi$ of positive $P$-periodic in $t$ functions $u(t, x)$ that vanish as $|x| \rightarrow \infty$ uniformly in $t$.

Lemma A.1. There exists a unique eigenvalue $\lambda$ of problem (A.1) whose corresponding eigenfunction $u \in \Xi$. This eigenvalue is real and of multiplicity one. Moreover, for every $s>0$ there is a constant $C_{s}$ such that $|u(t, z)| \leq C_{s}(1+|x|)^{-s}$. There is no other eigenvalue of problem (A.1) with an eigenfunction from $\Xi$.

Proof. Consider an auxiliary family of spectral problems

$$
-\frac{\partial u_{k}}{\partial t}+\mathcal{A}_{t} u_{k}=\lambda_{k} u_{k}, \quad t \in \mathbb{R},|x|<k, u_{k}(t+P, \cdot)=u_{k}(t, \cdot) \quad \text { and } u_{k}=0 \text { when }|x|=k .
$$

It follows from the Krein-Rutman theorem that for every $k>0$ the principal eigenvalue $\lambda_{k}$ (the eigenvalue with the largest real part) of this problem is real and simple, and that the corresponding eigenfunction $u_{k}$ is positive under a proper normalization. Moreover, using the Aronson estimates one shows that $\left|\lambda_{k}\right| \leq C$ with $C>0$ independent of $k$.

Normalizing the eigenfunctions $u_{k}$ of (A.2) by $u_{k}(0,0)=1$ and considering the uniform boundedness of $\left|\lambda_{k}\right|$ we derive by the Harnack inequality that for every $R_{0}>0$ it holds

$$
\sup \left\{\left|u_{k}(t, x)\right| ; x \in B_{R_{0}}, t \in \mathbb{R}\right\} \leq c\left(R_{0}\right)
$$

with $c\left(R_{0}\right)$ independent of $k$, where $B_{s}$ stands for the ball $\left\{x \in \mathbb{R}^{N} ;|x|<s\right\}$.

Consider now $v_{s}(x)=c\left(R_{0}\right)\left(R_{0} /|x|\right)^{s}$ with some $s>0$. Since $c(t, x)$ tends to $-\infty$ as $|x| \rightarrow \infty$, it is straightforward to check that $\left(\mathcal{A}_{t}-\lambda_{k}\right) v_{s}<0$ for $x \in\left(B_{k} \backslash B_{R_{0}}\right)$ if $R_{0}$ is large enough. By construction we also have $u_{k} \leq v_{s}$ when $x \in \partial\left(B_{k} \backslash B_{R_{0}}\right)$, this implies by the maximum principle that $u_{k} \leq v_{s}$ for $x \in\left(B_{k} \backslash B_{R_{0}}\right)$ (we assume that $R_{0}$ is so large that $c(t, x)-\lambda_{k} \leq 0$ for $\left.|x|>R_{0}\right)$. Passing to the limit as $k \rightarrow \infty$ possibly along a subsequence, we obtain that the limit function $u$ is a positive solution of the equation

$$
-\frac{\partial u}{\partial t}+\mathcal{A}_{t} u=\lambda u, \quad t \in \mathbb{R}, x \in \mathbb{R}^{N}
$$


with $u(0,0)=1$, and $\forall s>0$ there exists a constant $C_{s}$ such that $|u(t, x)| \leq C_{s}(1+|x|)^{-s}$. The proof of the simplicity of $\lambda$ is similar to that in [18].

To prove the uniqueness of $\lambda$ consider a family of adjoint equations

$$
\frac{\partial u_{k}^{*}}{\partial t}+A_{t}^{*} u_{k}^{*}=\lambda_{k} u_{k}^{*}, \quad t \in \mathbb{R}, x \in B_{k},
$$

subject to $P$-periodicity in $t$ condition and the Dirichlet condition $u^{*}=0$ for $x \in \partial B_{k}$. Again, by the Krein-Rutman theorem $u_{k}^{*}$ is positive. Moreover, since $\left|\lambda_{k}\right| \leq C$ with $C$ independent of $k$, $u_{k}^{*}$ satisfies the Harnack inequality uniformly in $k$. For the sake of definiteness we suppose that $u_{k}^{*}(0,0)=1$. Considering the uniform in $k$ Hölder continuity of $u_{k}^{*}$ (see [11], Thm. III.10.1), we conclude that $u_{k}^{*}$ converges, as $k \rightarrow \infty$, uniformly on compact sets in $[0, P] \times \mathbb{R}^{N}$, to a function $u^{*}$ being a positive solution to the equation

$$
\partial_{t} u^{*}+A_{t}^{*} u^{*}=\lambda u^{*}
$$

Suppose that there is another eigenvalue $\hat{\lambda} \neq \lambda$ of problem (A.1) such that the corresponding eigenfunction $\hat{u}$ is real and positive, and $\hat{u}(t, x) \rightarrow 0$ as $|x| \rightarrow \infty$ uniformly in $t \in[0, P]$. Adding if necessary a constant to $c(x, t)$, we can assume without loss of generality that $\lambda>0$ and $\hat{\lambda}>0$.

Let $R_{0}(s)$ be such that $A_{t}|x|^{-s}<0$ when $x \in \mathbb{R}^{N} \backslash B_{R_{0}}$. Then

$$
\begin{aligned}
\lambda_{k} \int_{0}^{P} \int_{B_{k} \backslash B_{R_{0}}}|x|^{-s} u_{k}^{*} \mathrm{~d} x \mathrm{~d} t & =\int_{0}^{P} \int_{B_{k} \backslash B_{R_{0}}}|x|^{-s}\left(\partial_{t}+A_{t}^{*}\right) u_{k}^{*} \mathrm{~d} x \mathrm{~d} t \\
& =\int_{0}^{P} \int_{B_{k} \backslash B_{R_{0}}} u_{k}^{*} A_{t}\left(|x|^{-s}\right) \mathrm{d} x \mathrm{~d} t+\int_{0}^{P} \int_{\partial B_{R_{0}}}|x|^{-s} \frac{\partial u_{k}^{*}}{\partial \nu_{a}} \mathrm{~d} \sigma \mathrm{d} t-\int_{0}^{P} \int_{\partial B_{R_{0}}} u_{k}^{*} \frac{\partial|x|^{-s}}{\partial \nu_{a}} \mathrm{~d} \sigma \mathrm{d} t \\
& +\int_{0}^{P} \int_{\partial B_{R_{0}}}(b(x, t) \cdot \nu) u_{k}^{*}|x|^{-s} \mathrm{~d} \sigma \mathrm{d} t+\int_{0}^{P} \int_{\partial B_{k}}|x|^{-s} \frac{\partial u_{k}^{*}}{\partial \nu_{a}} \mathrm{~d} \sigma \mathrm{d} t ;
\end{aligned}
$$

here $\nu$ stands for the unit exterior normal, and $\nu_{a}$ for the exterior conormal on $\partial\left(B_{k} \backslash B_{R_{0}}\right)$. By the standard elliptic estimates the second, third and forth terms on the right-hand side are bounded uniformly in $k$. Since the integral on the left-hand side is positive and the first and the last integral on the right-hand side are negative, this yields, after taking the limit as $k \rightarrow \infty$,

$$
\int_{0}^{P} \int_{\mathbb{R}^{N}} u^{*}(1+|x|)^{-s} \mathrm{~d} x \mathrm{~d} t<+\infty .
$$

Also we have the following pointwise bound for $\hat{u}$ whose proof is similar to that for $u, \forall s>0$ there is a constant $C_{s}$ such that $\hat{u}(t, x) \leq C_{s}(1+|x|)^{-s}$.

In order to complete the proof we choose $R_{0}>0$ such that $c(t, x)-\hat{\lambda} \leq 0$ when $|x| \geq R_{0}$ consider the function $\hat{v}_{k}$ which coincides with $\hat{u}$ in $\mathbb{R} \times B_{R_{0}}$ and is extended to $\mathbb{R} \times B_{k} \backslash B_{R_{0}}$ as the unique $P$-periodic in $t$ solution of the problem

$$
-\partial_{t} \hat{v}_{k}+A_{t} \hat{v}_{k}=\hat{\lambda} \hat{v}_{k}, \quad t \in \mathbb{R}, x \in B_{k} \backslash B_{R_{0}}, \hat{v}_{k}=\hat{u} \text { on } \partial B_{R_{0}}, \quad \hat{u}_{k}=0 \text { on } \partial B_{k} .
$$


We get, integrating by parts,

$$
\begin{aligned}
\hat{\lambda} \int_{0}^{P} \int_{B_{k}} \hat{v}_{k} u_{k}^{*} \mathrm{~d} x \mathrm{~d} t & =\int_{0}^{P} \int_{B_{R_{0}}} u_{k}^{*}\left(-\partial_{t} \hat{u}+A_{t} \hat{u}\right) \mathrm{d} x \mathrm{~d} t+\int_{0}^{P} \int_{B_{k} \backslash B_{R_{0}}} u_{k}^{*}\left(-\partial_{t} \hat{v}_{k}+A_{t} \hat{v}_{k}\right) \mathrm{d} x \mathrm{~d} t \\
& =\int_{0}^{P} \int_{B_{k}} \hat{v}_{k}\left(\partial_{t} u_{k}^{*}+A_{t}^{*} u_{k}^{*}\right) \mathrm{d} x \mathrm{~d} t+\int_{0}^{P} \int_{\partial B_{R_{0}}} u_{k}^{*}\left[\frac{\partial \hat{v}_{k}}{\partial \nu_{a}}\right] \mathrm{d} \sigma \mathrm{d} t \\
& =\lambda_{k} \int_{0}^{P} \int_{B_{k}} \hat{v}_{k} u_{k}^{*} \mathrm{~d} x \mathrm{~d} t+\int_{0}^{P} \int_{\partial B_{R_{0}}} u_{k}^{*}\left[\frac{\partial \hat{v}_{k}}{\partial \nu_{a}}\right] \mathrm{d} \sigma \mathrm{dt},
\end{aligned}
$$

where $\left[\frac{\partial \hat{v}_{k}}{\partial \nu_{a}}\right]$ denotes the jump of the conormal derivative of $\hat{v}_{k}$ through $\partial B_{R_{0}}$. Since $0 \leq \hat{v}_{k} \leq \hat{u}$ by the maximum principle, $\forall s>0$ we have $\left|\hat{v}_{k}(t, x)\right| \leq C_{s}(1+|x|)^{-s}$ uniformly in $k$. Using the maximum principle once more, we see that $\hat{v}_{k}$ converges uniformly to $\hat{u}$ as $k \rightarrow \infty$. Then by standard parabolic estimates $\left[\frac{\partial \hat{v}_{k}}{\partial \nu_{a}}\right] \rightarrow 0$ uniformly on $\mathbb{R} \times \partial B_{R_{0}}$. Thus passing to the limit $k \rightarrow \infty$ we obtain

$$
\hat{\lambda} \int_{0}^{P} \int_{\mathbb{R}^{N}} \hat{u} u^{*} \mathrm{~d} x \mathrm{~d} t=\lambda \int_{0}^{P} \int_{\mathbb{R}^{N}} \hat{u} u^{*} \mathrm{~d} x \mathrm{~d} t
$$

This contradicts the positiveness of $\hat{u}$.

Acknowledgements. This work was completed during the visit of V. Rybalko at the Narvik University College. He is indebted for the financial support and kind hospitality.

\section{REFERENCES}

[1] H. Abou-Kandil, G. Freiling, V. Ionescu and G. Jank, Matrix Riccati equations in control and systems theory. Systems and Control: Foundations and Applications. Birkhäuser Verlag, Basel (2003).

[2] G. Allaire and Y. Capdeboscq, Homogenization of a spectral problem in neutronic multigroup diffusion. Comput. Methods Appl. Mech. Engrg. 187 (2000) 91-117.

[3] G. Allaire and A. Piatnitski, Uniform spectral asymptotics for singularly perturbed locally periodic operators. Commun. Partial Differ. Eq. 27 (2002) 705-725.

[4] G. Allaire, I. Pankratova and A. Piatnitski, Homogenization and concentration for a diffusion equation with large convection in a bounded domain. J. Funct. Anal. 262 (2012) 300-330.

[5] G. Allaire and A.-L. Raphael, Homogenization of a convection-diffusion model with reaction in a porous medium. C. R. Math. Acad. Sci. Paris 344 (2007) 523-528.

[6] D.G. Aronson, Non-negative solutions of linear parabolic equations. Annal. Scuola Norm. Sup. Pisa 22 (1968) 607-694.

[7] Y. Capdeboscq, Homogenization of a diffusion equation with drift. C. R. Acad. Sci. Paris Ser. I Math. 327 (1998) 807-812.

[8] I. Capuzzo-Dolcetta and P.-L. Lions, Hamilton-Jacobi equations with state constraints. Trans. Amer. Math. Soc. 318 (1990) 643-683.

[9] P. Donato and A. Piatnitski, Averaging of nonstationary parabolic operators with large lower order terms. Multi scale problems and asymptotic analysis. Vol. 24, GAKUTO Int. Ser. Math. Sci. Appl. Gakkotosho, Tokyo (2006) 153-165.

[10] Yu. Kifer, On the principal eigenvalue in a singular perturbation problem with hyperbolic limit points and circles. J. Differ. Eqs. 37 (1980) 108-139.

[11] O.A. Ladyzhenskaya, V.A. Solonnikov and N.N. Uraltzeva, Linear and Quasi-linear Equations of Parabolic Type. AMS (1988).

[12] A. Piatnitski, Asymptotic Behaviour of the Ground State of Singularly Perturbed Elliptic Equations. Commun. Math. Phys. 197 (1998) 527-551.

[13] A. Piatnitski and V. Rybalko, On the first eigenpair of singularly perturbed operators with oscillating coefficients. Preprint available at www.arxiv.org, arXiv:1206.3754. 
[14] L.C. Evans and H. Ishii, A PDE approach to some asymptotic problems concerning random differential equation with small noise intensities. Ann. Inst. Henri Poincaré 2 (1985) 1-20.

[15] H. Mitake, Asymptotic solutions of Hamilton-Jacobi equations with state constraints. Appl. Math. Optim. 58 (2008) 393-410.

[16] H. Ishii and H. Mitake, Representation formulas for solutions of Hamilton-Jacobi equations with convex Hamiltonians. Indiana Univ. Math. J. 56 (2007) 2159-2183.

[17] M.H. Protter and H.F. Weinberger, On the spectrum of general second order operators. Bull. Amer. Math. Soc. 72 (1966) $251-255$.

[18] A.L. Pyatnitskii and A.S. Shamaev, On the asymptotic behavior of the eigenvalues and eigenfunctions of a nonselfadjoint operator in $\mathbb{R}^{n}$. (Russian) Tr. Semin. Im. I.G. Petrovskogo 23 (2003) 287-308, 412; translation in J. Math. Sci. 120 (2004) $1411-1423$.

[19] M.I. Freidlin and A.D. Wentzell, Random perturbations of dynamical systems, vol. 260. Fundamental Principles Math. Sci. Springer-Verlag, New York (1984). 